\title{
Wavelets by orthogonal rational kernels
}

\author{
Adhemar Bultheel and Pablo González-Vera \\ Dedicated to Jerry Lange on the occasion of his 70th birthday
}

\begin{abstract}
Let $\mathcal{L}_{n}$ be the space of polynomials or rational functions of degree $n$ at most with poles in a prescribed set of numbers inside the unit disk. Consider a complex inner product with respect to a finite positive measure on the unit circle. Suppose $\left\{\phi_{k}\right\}_{k=0}^{n}$ is the corresponding orthonornal basis for $\mathcal{L}_{n}$. Set $n=2^{s}$ and $\mathcal{V}_{s}=\mathcal{L}_{n}$. Then $k_{n}(z, w)=\sum_{k=0}^{n} \phi_{k}(z) \overline{\phi_{k}(w)}$, is a reproducing kernel for $\mathcal{V}_{s}$. For fixed $w$, such reproducing kernels are known to be functions localized in the neighborhood of $z=w$. Moreover, by an appropriate choice of the parameters $\left\{\xi_{n k}\right\}_{k=0}^{n}$, the functions $\left\{\varphi_{n, k}(z)=k_{n}\left(z, \xi_{n k}\right)\right\}_{k=0}^{n}$ will be an orthogonal basis for $\mathcal{V}_{s}$. The orthogonal complement $\mathcal{W}_{s}=\mathcal{V}_{s+1} \ominus \mathcal{V}_{s}$ is spanned by the functions $\left\{\psi_{n, k}(z)=l_{n}\left(z, \eta_{n k}\right)\right\}_{k=0}^{n-1}$ for an appropriate choice of the parameters $\left\{\eta_{n k}\right\}_{k=0}^{n-1}$ where $l_{n}=k_{2 n}-k_{n}$ is the reproducing kernel for $\mathcal{W}_{s}$. These observations form the basic ingredients for the construction of rational wavelets on the unit circle with respect to an arbitrary positive measure.
\end{abstract}

\section{Introduction}

The main motivation for this paper is the basic observation that was made by Fischer and Prestin [FP97] in the case of polynomials on the real line.

This observation is that a reproducing kernel in a reproducing kernel Hilbert space has the property that it is a localized function. If $\mathcal{H}$ is a Hilbert space of functions defined on some set $\mathbb{X} \subset \mathbb{C}$, then it is called a reproducing kernel Hilbert space if there is a kernel function $k_{w} \in \mathcal{H}(w \in \mathbb{X}$ is a parameter $)$ such that $\left\langle f, k_{w}\right\rangle=f(w)$ for all $f \in \mathcal{H}, w \in \mathbb{X}$. It has the remarkable property that it gives the solution of $\inf _{f \in \mathcal{H}}\{\|f\|: f(w)=1, w \in \mathbb{X}\}$ namely $f=k_{w} / k_{w}(w)$. This can be interpreted as follows: without the constraint $f(w)=1$, the solution would be $f \equiv 0$. The constraint forces $f$ to take the value 1 at $w$, but the optimization problem implies that in $\mathbb{X} \backslash\{w\}$, the function $f$ is "as small as possible". If the

1991 Mathematics Subject Classification. Primary 42C05; Secondary 46E22, 42A38.

The work of the first author was performed as part of the project "Orthogonal systems and their applications" of the FWO under grant \#G0278.97.

The work of the second author was supported by the scientific research project of the Spanish D.G.I.C.Y.T. under contract PB96-1029. 
space $\mathcal{H}$ consists of "smooth" functions like polynomials or rationals, then $f$ has typically a "bump" in $z=w$ and much smaller oscillations away from $z=w$.

Fischer and Prestin exploited this fact to construct polynomial wavelets. Consider the space of polynomials on an interval (possibly infinite) $[a, b] \subseteq \mathbb{R}$ equiped with the inner product $\langle p, q\rangle=\int_{a}^{b} p(x) q(x) d \mu(x)$ where $\mu$ is a finite positive measure. The subspaces $\mathcal{L}_{n}$ of polynomials of degree at most $n$ form a nested sequence $\mathcal{L}_{0} \subset \mathcal{L}_{1} \subset \cdots \subset \mathcal{L}_{n} \subset \cdots$ of subspaces. If $f_{n}$ is the projection of some $f \in L_{2}(\mu, \mathbb{R})$ onto $\mathcal{L}_{n}$, it is clear that for increasing $n$ we shall get better and better approximations for $f$, i.e., more and more details of $f$ will become visible in the projections $f_{n}$. One may say that the $\mathcal{L}_{n}$ represent different scales or resolution levels of the space $L_{2}(\mu, \mathbb{R})$.

More generally, in multiresolution analysis, one decomposes a function space in several resolution levels and the idea is to represent the functions from that function space by a low resolution approximation and adding to it the successive details that lift it to resolution levels of increasing detail.

Wavelet analysis couples the multiresolution idea with a special choice of bases for the different resolution spaces and for the wavelet spaces that represent the difference between successive resolution spaces. Thus if $\mathcal{V}_{s}$ are the resolution spaces: $\mathcal{V}_{0} \subset \mathcal{V}_{1} \subset \cdots$, then the wavelet spaces are given by the differences: $\mathcal{W}_{s}=\mathcal{V}_{s+1} \ominus \mathcal{V}_{s}$

By their definition, the functions in $\mathcal{W}_{s}$ will automatically be band-limited in their detail information. This means that the basis functions for these $\mathcal{W}_{s}$ (which are called wavelet functions) will have Fourier transforms that mainly live on a relatively small interval i.e., they are small outside that interval. Moreover, for true wavelet functions, one wants the functions themselves to live mainly on a small interval as well. Then a local change in the function $f$ will only have considerable effect on the components along the basis functions that have their main part in the neighborhood of the change and it will much less affect the other components. Conversely, changing one component will only have a local effect on the function.

The kernel functions which we described above do have this wavelet-like behavior and the problem is thus whether it is possible to give a basis of reproducing kernels that span the successive resolution spaces and wavelet spaces. The answer to this question is affirmative and is completely analyzed in $\left[\right.$ FP97] for $L_{2}(\mu, \mathbb{R})$ where the subspaces are spanned by polynomials.

In this paper we give two modifications of this idea. First, we consider complex function spaces where orthogonality is with respect to a measure on the complex unit circle and in the second place we generalize the space of polynomials to the space of rational functions whose poles are in a certain prescribed set. To be more precise, $\mathcal{L}_{n}$ will be the space of functions of the form $p_{n} / \pi_{n}$ where $p_{n}$ is a polynomial of degree at most $n$ and $\pi_{n}(z)=\prod_{k=1}^{n}\left(1-\bar{\alpha}_{n} z\right)$. We assume that all the $\alpha_{k}$ are inside the open unit disk. A parallel theory could have been developed where all the $\alpha_{k}$ are on the unit circle, but we shall not do this here. A similar discussion for rational wavelets on the real line with all the poles on the real line was given in [BGV98].

The results presented in this paper rely on two pilars: the theory of wavelets, which has to be generalized at different places, very much like it was done in [FP97] and the theory of orthogonal rational functions on the unit circle.

The plan of the paper is as follows. First we introduce the rational function spaces we shall work with and give the basic ideas of multiresolution analysis. In 
Section 4 we summarize the main properties of reproducing kernels and establish a way to make them produce a basis. In the next two sections we discuss orthogonal basis functions. First the basis of orthogonal rational functions (ORF) and in the next section it is shown how one can find a basis of orthogonal reproducing kernels (ORK). In Section 7 gives a discussion of how to construct a wavelet basis of reproducing kernels (WRK), but it is not obvious how this can be made orthogonal in a general case. Section 8 describes the dual (i.e., biorthogonal) basis functions and this is used in the next section to give the explicit formulas for analysis and synthesis computations. Next, the generalization of multiresolution analysis (MRA) and completeness properties are given and some theorems about the symmetry of the wavelets and scaling functions. Finally, in Section 12, some computational aspects are discussed.

\section{The rational functions}

We consider the complex Hilbert space $L_{2}=L_{2}(\mathbb{T}, \mu)$ of functions which are square integrable on the unit circle $\mathbb{T}=\{z \in \mathbb{C}:|z|=1\}$ with respect to some finite positive measure $\mu$. The inner product is given by

$$
\langle f, g\rangle=\int_{\mathbb{T}} f(t) \overline{g(t)} \mathrm{d} \mu(t) .
$$

For simplicity we assume that $\int_{\mathbb{T}} \mathrm{d} \mu=1$. Denote by $\Pi_{n}$ the set of polynomials of degree at most $n$, then for a given sequence of points $\alpha_{1}, \alpha_{2}, \ldots$ all inside the unit disk $\mathbb{D}=\{z \in \mathbb{C}:|z|<1\}$, we consider the spaces of rational functions

$$
\mathcal{L}_{n}=\left\{\frac{p_{n}}{\pi_{n}}: p_{n} \in \Pi_{n}\right\}, \quad n=0,1, \ldots
$$

where $\pi_{0}=1$ and $\pi_{n}=\prod_{k=1}^{n}\left(1-\bar{\alpha}_{k} z\right), n=1,2, \ldots$ We refer to the "polynomial case" as corresponding to the choice $\alpha_{k}=0$ for all $k=1,2, \ldots$ In the polynomial case, all the poles are at infinity and we have $\Pi_{n}=\mathcal{L}_{n}$.

An obvious choice to represent these spaces is to construct an orthonormal basis, i.e., a sequence of functions $\left\{\phi_{0}, \phi_{1}, \ldots\right\}$ such that $\phi_{n} \in \mathcal{L}_{n} \backslash \mathcal{L}_{n-1}, \phi_{n} \perp \mathcal{L}_{n-1}$ and $\left\|\phi_{n}\right\|=1$. Such functions were first considered by M.M. Djrbashian in the sixties (see the summary [Djr90]) and later by A. Bultheel, K. Pan, X. Li, and in a series of papers by E. Hendriksen and $\mathrm{O}$. Njasstad in collaboration with the authors. Most of the results we need are found in one of these papers, but we do not reference all of them. Instead we refer to a forthcoming monograph [BGVHN98b] which brings together all these results in a systematic way, or to a preliminary report [BGVHN90] which collects many of the properties needed.

\section{Wavelet transform: decomposition and reconstruction}

As explained in the introduction, we consider a multiresolution structure defined by nested subspaces $\mathcal{L}_{n}$ for increasing $n$. More precisely, we consider the spaces $\mathcal{V}_{s}=\mathcal{L}_{2^{s}}$ for $s=0,1, \ldots$ Thus the resolution spaces $\mathcal{V}_{s}$ have dimension $2^{s}+1$ $s \geq 0$. We define the wavelet spaces $\mathcal{W}_{s}$ as the orthogonal complement of $\mathcal{V}_{s}$ in $\mathcal{V}_{s+1}$, thus $\mathcal{W}_{s}=\mathcal{V}_{s+1} \ominus \mathcal{V}_{s}$. The dimension of $\mathcal{W}_{s}$ is $2^{s}$. To simplify the notation we shall henceforth assume that $n=2^{s}$ and work with the resolution spaces $\mathcal{L}_{n}$ and the wavelet spaces $\mathcal{K}_{n}=\mathcal{L}_{2 n} \ominus \mathcal{L}_{n}$. To get started, we need the initial spaces $\mathcal{V}_{-1}=\mathcal{L}_{0}$ and $\mathcal{W}_{-1}=\mathcal{K}_{0}=\mathcal{L}_{1} \ominus \mathcal{L}_{0}$. Both have dimension 1. 
REMARK 3.1. We could avoid this special treatment of the initial spaces by defining $\mathcal{V}_{s}=\mathcal{L}_{2^{s}-1}$, and $\mathcal{W}_{s}=\mathcal{V}_{s+1} \ominus \mathcal{V}_{s}$ for $s=0,1, \ldots$ Then $\mathcal{V}_{s}$ and $\mathcal{W}_{s}$ both have dimension $2^{s}$. However, since the previously mentioned choice was made in [FP97], we keep the same kind of convention in this text.

The projection $f_{n}$ of $f \in L_{2}$ onto $\mathcal{L}_{n}=\mathcal{V}_{s}$ is called the representation of $f$ at scale or resolution level $s$. For every scale $s$, we can write $f_{2 n}=f_{n}+g_{n}$ where $f_{2 n} \in \mathcal{L}_{2 n}, f_{n} \in \mathcal{L}_{n}$, and $g_{n} \in \mathcal{K}_{n}$. The projection $f_{n}$ is the low resolution part and $g_{n}$ is the high resolution part of $f_{2 n}$. The low resolution part is decomposed again as $g_{n}=f_{n / 2}+g_{n / 2}$ etc. Thus, after a number of such decompositions we find

$$
f_{2 n}=f_{0}+g_{0}+\sum_{k=0}^{s} g_{2^{k}} .
$$

Thus

$$
\mathcal{V}_{s+1}=\mathcal{V}_{-1} \oplus \mathcal{W}_{-1} \oplus \mathcal{W}_{0} \oplus \cdots \oplus \mathcal{W}_{t} \oplus \cdots \oplus \mathcal{W}_{s}
$$

or equivalently

$$
\mathcal{L}_{2 n}=\mathcal{L}_{0} \oplus \mathcal{K}_{0} \oplus \mathcal{K}_{1} \oplus \cdots \oplus \mathcal{K}_{2^{t}} \oplus \cdots \oplus \mathcal{K}_{n}
$$

Now suppose that for $n=2^{s}, s=0,1, \ldots$, the wavelet spaces $\mathcal{K}_{n}$ are spanned by localized basis functions (wavelet functions) $\left\{\psi_{n, k}: k=0, \ldots, n-1\right\}$ and the resolution spaces $\mathcal{L}_{n}$ are spanned by localized basis functions (scaling functions) $\left\{\varphi_{n, k}: k=0,1, \ldots, n\right\}$.

If we suppose that $f_{n}=\sum_{k=0}^{n} p_{n, k} \varphi_{n, k}$ and $g_{n}=\sum_{k=0}^{n-1} q_{n, k} \psi_{n, k}$, then we can write

$$
f_{2 n}=p_{0,0} \varphi_{0,0}+\sum_{k=0}^{s} \sum_{l=0}^{2^{k}-1} q_{2^{k}, l} \psi_{2^{k}, l} .
$$

The set of coefficients $\left\{p_{0,0}\right\} \cup\left\{q_{2^{k}-1, l}: k=0, \ldots, s ; l=0, \ldots, 2^{k}-1\right\}$ is called the (discrete) wavelet transform of $f_{2 n}$.

Let us introduce the notation for any integer $n \geq 0$

$$
\boldsymbol{p}_{n}=\left[p_{n, 0}, \ldots, p_{n, n}\right] \quad \text { and } \quad \boldsymbol{\varphi}_{n}=\left[\varphi_{n, 0}, \ldots, \varphi_{n, n}\right]^{T}
$$

and

$$
\boldsymbol{q}_{n}=\left[q_{n, 0}, \ldots, q_{n, n-1}\right] \quad \text { and } \quad \boldsymbol{\psi}_{n}=\left[\psi_{n, 0}, \ldots, \psi_{n, n-1}\right]^{T}
$$

(the superscript ${ }^{T}$ means transpose) so that $f_{n}=\boldsymbol{p}_{n} \boldsymbol{\varphi}_{n}$ and $g_{n}=\boldsymbol{q}_{n} \boldsymbol{\psi}_{n}$. In one step of the analysis or decomposition algorithm one starts from the coefficients $\boldsymbol{p}_{2 n}$ and transforms this in $\boldsymbol{p}_{n}$ and $\boldsymbol{q}_{n}$, which corresponds to a change of basis like

$$
f_{2 n}=\sum_{k=0}^{2 n} p_{2 n, k} \varphi_{2 n, k}=\sum_{k=0}^{n} p_{n, k} \varphi_{n, k}+\sum_{k=0}^{n-1} q_{n, k} \psi_{n, k}
$$

or more compactly: $f_{2 n}=\boldsymbol{p}_{2 n} \boldsymbol{\varphi}_{2 n}=\boldsymbol{p}_{n} \boldsymbol{\varphi}_{n}+\boldsymbol{q}_{n} \boldsymbol{\psi}_{n}$. In the next step one transforms $\boldsymbol{p}_{n}$ into $\boldsymbol{p}_{n / 2}$ and $\boldsymbol{q}_{n / 2}$ etc. The synthesis or reconstruction algorithm goes in the opposite direction and generates $\boldsymbol{p}_{2 n}$ from $\boldsymbol{p}_{n}$ and $\boldsymbol{q}_{n}$. Thus it is important that we have an efficient algorithm to do the transformations back and forth between $\boldsymbol{p}_{2 n} \leftrightarrow\left\{\boldsymbol{p}_{n}, \boldsymbol{q}_{n}\right\}$.

Such a transformation should preferably be unitary for the ease of inversion and for the numerical stability. Thus the basis $\left\{\varphi_{2 n, k}: k=0, \ldots, 2 n\right\}$ as well as 
the basis $\left\{\varphi_{n, k}: k=0, \ldots, n\right\} \cup\left\{\psi_{n, k}: k=0, \ldots, n-1\right\}$ should be orthogonal. By construction, the $\psi_{n, k}$ are orthogonal to the $\varphi_{n, l}$ and hence to all $\varphi_{m, l}$ and $\psi_{m, l}$ with $m<n$.

An obvious choice for the basis functions would be the orthogonal rational functions (ORF) $\phi_{n}$ that we have defined at the end of Section 2. Thus $\varphi_{n, k}=\phi_{k}$ for $k=0, \ldots, n$ and $\psi_{n, k}=\phi_{n+1+k}$ for $k=0, \ldots, n-1$. However, except for very special cases, these functions are not localized and therefore are not wavelet-like. Because reproducing kernels do have this property, we shall try to introduce a basis of orthogonal rational kernel (ORK) functions.

\section{Reproducing kernels}

The following properties concerning reproducing kernels are well known (see for example [Aro50, Mes62, Don74])

THEOREM 4.1. We consider an m-dimensional subspace $\mathcal{K}$ of $\mathcal{L}_{n}$. Assume that its reproducing kernel is denoted as $k(z, w)$. Then

1. For any orthonormal basis $\left\{e_{1}, e_{2}, \ldots, e_{m}\right\}$ of $\mathcal{K}$, the reproducing kernel is given by

$$
k(z, w)=\sum_{k=1}^{m} e_{k}(z) \overline{e_{k}(w)} .
$$

2. For any set of distinct points $\left\{w_{1}, w_{2}, \ldots, w_{m}\right\}$ among the points of analyticity for $\mathcal{K}$,

$$
\left\langle k\left(z, w_{j}\right), k\left(z, w_{i}\right)\right\rangle=k\left(w_{i}, w_{j}\right)
$$

and the matrix

$$
M=\left[k\left(w_{i}, w_{j}\right)\right]_{i, j=1}^{m}
$$

is positive semi-definite.

3. The orthogonal projection of $f \in \mathcal{L}_{n}$ onto $\mathcal{K}$ is given by

$$
P_{\mathcal{K}} f=\langle f(z), k(z, w)\rangle \text {. }
$$

4. For any point $w$ where it makes sense,

$$
\inf _{f \in \mathcal{K}}\{\|f\|: f(w)=1\}=[k(w, w)]^{-1}
$$

and the minimizer is $f(z)=k(z, w) / k(w, w)$.

Also the following lemma is easy to verify.

Lemma 4.2. Let $\left\{\phi_{0}, \ldots \phi_{n}\right\}$ be the orthonormal basis for $\mathcal{L}_{n}$ as introduced above, then for any set of distinct points $\boldsymbol{x}=\left\{x_{0}, x_{1}, \ldots, x_{n}\right\}$ which are points of analyticity for functions in $\mathcal{L}_{n}$, the matrix

$$
\Phi_{n}(\boldsymbol{x})=\left[\begin{array}{ccc}
\phi_{0}\left(x_{0}\right) & \cdots & \phi_{0}\left(x_{n}\right) \\
\vdots & & \vdots \\
\phi_{n}\left(x_{0}\right) & \cdots & \phi_{n}\left(x_{n}\right)
\end{array}\right]
$$

is regular.

Proof. This is obvious because the $\phi_{k}$ form a Haar system in the polynomial as well as in the rational case. See Davis [Dav63, Chap. II, Sect. 2.4]. 
FiguRE 1. The real (left) and imaginary part (right) of the functions $k_{n}(z, w)=\sum_{k=0}^{n}(z / w)^{k}($ top $)$ and $l_{n}(z, w)=\sum_{k=n+1}^{2 n}(z / w)^{k}$ (bottom) for $z=e^{i \theta}$ and $w=1$, plotted as functions of $\theta$. Note that the real parts are even functions, while the imaginary parts are odd functions.

A: $n=4$
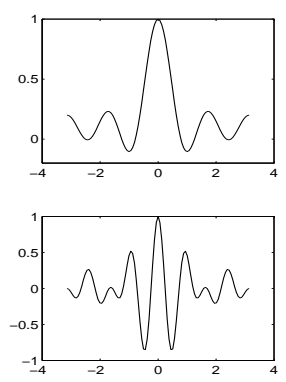
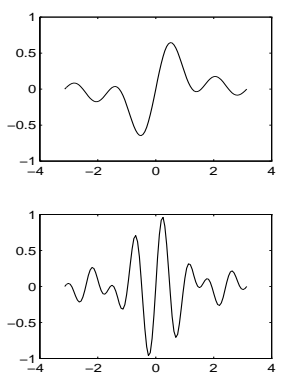

B: $n=8$
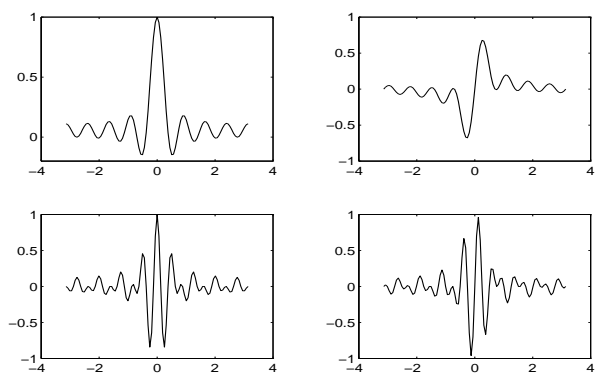

An immediate consequence is that we can choose a basis of reproducing kernels for the spaces $\mathcal{L}_{n}$.

Corollary 4.3. If $k_{n}(z, w)$ is the reproducing kernel for $\mathcal{L}_{n}$ and the $\boldsymbol{x}=$ $\left\{x_{k}\right\}_{k=0}^{n}$ are $n+1$ distinct points of analyticity for $\mathcal{L}_{n}$, then the functions $\left\{\varphi_{n j}(z)=\right.$ $\left.k_{n}\left(z, x_{j}\right)\right\}_{j=0}^{n}$ form a basis for $\mathcal{L}_{n}$.

Proof. Since, with the matrix $\Phi_{n}(\boldsymbol{x})$ as in the previous lemma and denoting by a superscript ${ }^{H}$ the complex conjugate transpose:

$$
\left[\begin{array}{c}
k_{n}\left(z, x_{0}\right) \\
\vdots \\
k_{n}\left(z, x_{n}\right)
\end{array}\right]=\Phi_{n}^{H}(\boldsymbol{x})\left[\begin{array}{c}
\phi_{0}(z) \\
\vdots \\
\phi_{n}(z)
\end{array}\right]
$$

the statement follows because $\left\{\phi_{k}\right\}_{k=0}^{n}$ is a basis and $\Phi_{n}(\boldsymbol{x})$ is regular.

EXAMPLE 4.1. Let us give a very simple example. Consider the polynomial case, i.e., all $\alpha_{k}=0$ and assume that the measure $\mu$ is the normalized Lebesgue measure on $\mathbb{T}$. Then the orthonormal polynomials are just $\phi_{k}(z)=z^{k}$. This is the basis used in classical Fourier analysis. Each basis function has a Fourier transform which is a Dirac impulse and is thus extremely localized, but the functions themselves are in modulus equal to 1 everywhere on $\mathbb{T}$ and thus they have the worst possible localization on $\mathbb{T}$. However the kernels $k_{2 n}(z, w)=\sum_{k=0}^{2 n} z^{k} \bar{w}^{k}$ are

$$
k_{2 n}(z, w)=\left(\frac{z}{w}\right)^{n} \sum_{k=-n}^{n}\left(\frac{z}{w}\right)^{k}=e^{i n(\theta-\omega)} \frac{\sin \left(n+\frac{1}{2}\right)(\theta-\omega)}{\sin \frac{1}{2}(\theta-\omega)}, \quad z=e^{i \theta}, w=e^{i \omega} .
$$

Hence these kernels are complex exponentials modulated by the Dirichlet kernel, which is localized around $\theta=\omega$. This can be verified in Figure 1. For the spaces $\mathcal{K}_{n}=\mathcal{L}_{2 n} \ominus \mathcal{L}_{n}$, the reproducing kernels are given by $l_{n}(z, w)=k_{2 n}(z, w)-$ $k_{n}(z, w)=\sum_{k=n+1}^{2 n} \phi_{k}(z) \overline{\phi_{k}(w)}$. In our example this is just $(z \bar{w})^{n+1} \sum_{k=0}^{n-1}(z \bar{w})^{k}$. The plots for this trivial example are given in the bottom rows of Figure 1. It 
is immediately observed that these oscillate more but otherwise have properties similar to the kernels for $\mathcal{L}_{n}$.

Although we have plotted the kernel $l_{n}$ for the space $\mathcal{K}_{n}$ in the previous example, it is not as trivial as for the $k_{n}$ to conclude that there are numbers $\left\{y_{0}, \ldots, y_{n-1}\right\}$ such that $\left\{l_{n}\left(z, y_{k}\right)\right\}_{k=0}^{n-1}$ will form a basis for $\mathcal{K}_{n}$. Indeed, the system $\left\{\phi_{k}\right\}_{k=n+1}^{2 n}$ will not be a Haar system in general. Before we investigate this any further, we shall first recall several properties of the ORF basis $\left\{\phi_{k}\right\}$ in the next section.

\section{The ORF basis}

We need several properties of the orthogonal rational functions (ORF) $\phi_{n}$ that were defined in Section 2. Almost all of them can be found in [BGVHN98b]. We shall only give a proof of those properties that are new.

We start by giving some notation. Besides the points $\left\{\alpha_{1}, \alpha_{2}, \ldots\right\} \subset \mathbb{D}$, we define the special point $\alpha_{0}=0$. Furthermore, we define for $k=1,2, \ldots$ the Blaschke factors

$$
\zeta_{k}(z)=z_{k} \frac{z-\alpha_{k}}{1-\bar{\alpha}_{k} z}, \quad \text { with } z_{k}=1 \text { if } \alpha_{k}=0 \text { and } z_{k}=-\frac{\bar{\alpha}_{k}}{\left|\alpha_{k}\right|} \text { otherwise, }
$$

and the Blaschke products

$$
B_{0}=1, \quad B_{n}=\zeta_{1} \cdots \zeta_{n}, \quad n=1,2, \ldots
$$

For any function $f$ we set $f_{*}(z)=\overline{f(1 / \bar{z})}$ and if $f_{n} \in \mathcal{L}_{n}$ (it should be clear from the context what space $\mathcal{L}_{n}$ is involved) we define $f_{n}^{*}(z)=B_{n}(z) f_{*}(z)$.

Obviously $\mathcal{L}_{n}=\operatorname{span}\left\{B_{0}, B_{1}, \ldots, B_{n}\right\}$. If we write $\phi_{n}=a_{n 0} B_{0}+a_{n 1} B_{1}+\cdots+$ $a_{n n} B_{n}$, then the orthonormal $\phi_{n}$ are uniquely defined if we impose the normalization $\kappa_{n}=\phi_{n}^{*}\left(\alpha_{n}\right)>0$. (Note that $\kappa_{n}$ is the $a_{n n}$ from the previous expansion.) We shall use the notation $\phi_{n}$ to mean this uniquely defined orthonormal rational function.

The ORF satisfy a recurrence relation that generalizes the Szegö recurrence relation for orthogonal polynomials on the unit circle. In fact the polynomials form a special case obtained by setting all $\alpha_{k}=0$.

THEOREM 5.1. For the orthonormal basis functions $\phi_{k}=\kappa_{k} B_{k}+\cdots, \kappa_{k}>0$ in $\mathcal{L}_{n}$, a recursion of the following form exists

$$
\phi_{n}(z)=e_{n} \eta_{n 1}\left[\frac{z-\alpha_{n-1}}{1-\bar{\alpha}_{n} z} \phi_{n-1}(z)+\bar{\lambda}_{n} \frac{1-\bar{\alpha}_{n-1} z}{1-\bar{\alpha}_{n} z} \phi_{n-1}^{*}(z)\right]
$$

and its superstar conjugate is

$$
\phi_{n}^{*}(z)=e_{n} \eta_{n 2}\left[\lambda_{n} \frac{z-\alpha_{n-1}}{1-\bar{\alpha}_{n} z} \phi_{n-1}(z)+\frac{1-\bar{\alpha}_{n-1} z}{1-\bar{\alpha}_{n} z} \phi_{n-1}^{*}(z)\right] .
$$

The parameter $\lambda_{n}$ is given by

$$
\lambda_{n}=-\frac{\left\langle\phi_{k}(z), \frac{z-\alpha_{n-1}}{1-\bar{\alpha}_{n} z} \phi_{n-1}(z)\right\rangle}{\left\langle\phi_{k}(z), \frac{1-\bar{\alpha}_{n-1} z}{1-\bar{\alpha}_{n} z} \phi_{n-1}^{*}(z)\right\rangle}=z_{n} \frac{1-\bar{\alpha}_{n-1} \alpha_{n}}{1-\bar{\alpha}_{n} \alpha_{n-1}} \frac{\overline{\phi_{n}\left(\alpha_{n-1}\right)}}{\phi_{n}^{*}\left(\alpha_{n-1}\right)}
$$

where $0 \leq k \leq n-1$. 
The constant $e_{n}>0$ defines the modulus and it is given as the positive square root of

$$
e_{n}^{2}=\frac{1-\left|\alpha_{n}\right|^{2}}{1-\left|\alpha_{n-1}\right|^{2}} \frac{1}{1-\left|\lambda_{n}\right|^{2}}
$$

(Note $\left|\lambda_{n}\right|<1$.)

The $\eta_{n 1}$ and $\eta_{n 2}$ are constants on $\mathbb{T}$ which arrange for $\kappa_{n}=\phi_{n}^{*}\left(\alpha_{n}\right)>0$. They are related by $\eta_{n 2}=z_{n} \eta_{n 1}$.

The initial conditions are $\phi_{0}=\phi_{0}^{*}=1$.

For the development of the theory of rational wavelets that we give in the next sections, the precise recursion is irrelevant. However, when performing the proper computations, the availability of a recurrence relation is really important, since a general Gram-Schmidt procedure to compute the ORF basis is unacceptable, because of all the inner products (i.e., integrals) to be evaluated.

In this perspective it is important to have good recurrence relations for the ORF basis, or even for the reproducing kernels (see later). The above recurrence is not the only one that one could use. We give some samples of theoretically equivalent recursions, which may have a quite different numerical behaviour. These recurrences are all related to continued fractions.

For example, the previous recurrences are related to a continued fraction of the form

$$
1-\frac{1}{{ }_{2}}+\frac{a_{1}}{b_{1}}+\frac{a_{2}}{\not b_{2}}+\cdots
$$

where

$$
\begin{gathered}
a_{2 k+1}=\frac{z-\alpha_{k}}{1-\bar{\alpha}_{k} z}, \quad b_{2 k+1}=\bar{\lambda}_{k+1} \\
a_{2 k}=\bar{\eta}_{k 1} \eta_{k 2}\left(1-\left|\lambda_{k}\right|^{2}\right), \quad b_{2 k}=\bar{\eta}_{k 1} \eta_{k 2} \lambda_{k}
\end{gathered}
$$

Such a continued fraction is (equivalent to) a Perron-Carathéodory (or PC) fraction when all $\alpha_{k}=0$ and it is called a Nevanlinna-Pick (or NP) fraction in the general case because it is related to the solution of the Nevanlinna-Pick interpolation problem (see Section 12). The successive convergents are

$$
\frac{\hat{\phi}_{0}}{\phi_{0}},-\frac{\hat{\phi}_{0}^{*}}{\phi_{0}^{*}}, \ldots, \frac{\hat{\phi}_{n}}{\phi_{n}},-\frac{\hat{\phi}_{n}^{*}}{\phi_{n}^{*}}, \ldots
$$

where the $\phi_{k}$ are the ORF and the $\hat{\phi}_{k}$ are the so called associated functions or functions of the second kind.

The successive approximants are interpolating functions for the Riesz-Herglotz transform of the measure:

$$
\Omega(z)=\int_{\mathbb{T}} \frac{t+z}{t-z} \mathrm{~d} \mu(t)
$$

in the sense that

$$
\Omega(z)+\frac{\hat{\phi}_{n}(z)}{\phi_{n}(z)}=\frac{\hat{h}(z)}{z B_{n}(z)} \quad \text { and } \quad \Omega(z)-\frac{\hat{\phi}_{n}^{*}(z)}{\phi_{n}^{*}(z)}=z B_{n}(z) h(z)
$$

with $\hat{h}(z)$ analytic in $|z|>1$ while $h(z)$ is analytic in $|z|<1$.

Several variants of this continued fraction can be obtained for example by applying several equivalence transforms or contractions. Alternatively, they may be 
derived from the recurrence relations obtained by eliminating some terms from the coupled recursions (5.1-5.2) given in the previous theorem. For example, one could eliminate $\phi_{n-1}^{*}$ from (5.1) which would give a relation between $\phi_{n}, \phi_{n}^{*}$ and $\phi_{n-1}$. We shall not mention all of them. Several possibilities are given in [BGVHN98b].

The most prominent one of the alternatives is some variant of a three-term recurrence relation, which can be written in the form

$$
\begin{aligned}
\bar{\lambda}_{k} f_{k+1}(z)= & {\left[\zeta_{k}(z) \bar{\lambda}_{k}+\eta_{k 2} \bar{\eta}_{k 1} \bar{\lambda}_{k+1}\right] f_{k}(z)-} \\
& {\left[\zeta_{k-1}(z) \eta_{k 2} \bar{\eta}_{k 1} \bar{\lambda}_{k+1}\left(1-\left|\lambda_{k}\right|^{2}\right)\right] f_{k-1}(z) . }
\end{aligned}
$$

The initial conditions

$$
f_{0}=1, \quad f_{1}=e_{1} \eta_{1,1} 1 \frac{z+\bar{\lambda}_{1}}{1-\bar{\alpha}_{1} z}
$$

will give $f_{k}=\phi_{k}$. The other solution is given by functions of the second kind.

In the polynomial case, the continued fraction corresponding to this recurrence is related to an M-fraction and the corresponding recurrence for the $\phi_{n}^{*}$ is related to T-fractions. The convergents of these continued fractions are related to two-point Padé approximants. In the general case these become multipoint Padé approximants and the continued fractions are sometimes called MP-fractions. Many more of these continued fractions related to ORF are described in [BGVHN98a].

As we have seen, it is not exactly the ORF basis that we want to use, but the basis of reproducing kernels. Of course, once we know the ORF basis, we can compute the reproducing kernel basis, but it would be much more interesting if we had a recurrence directly for the reproducing kernels. Such a recurrence does indeed exist. In fact it are even simpler to formulate.

THEOREM 5.2. Let $k_{n}(z, w)$ be the reproducing kernels for the spaces $\mathcal{L}_{n}$. Then

$$
k_{n}(z, w)=c_{n}\left[\left(\rho_{n} \zeta_{n}(z)+\gamma_{n}\right) k_{n-1}^{*}(z, w)+\left(\rho_{n} \zeta_{n}(z) \bar{\gamma}_{n}+1\right) k_{n-1}(z, w)\right]
$$

and

$$
k_{n}^{*}(z, w)=c_{n}\left[\left(\zeta_{n}(z)+\bar{\rho}_{n} \gamma_{n}\right) k_{n-1}^{*}(z, w)+\left(\zeta_{n}(z) \bar{\gamma}_{n}+\bar{\rho}_{n}\right) k_{n-1}(z, w)\right]
$$

where

$$
\begin{aligned}
& \rho_{n}=\rho_{n}(w)=\overline{\phi_{n}(w)} / \phi_{n}^{*}(w) \\
& \gamma_{n}=\gamma_{n}(w)=-\zeta_{n}(w) \rho_{n}(w) \\
& c_{n}=c_{n}(w)=\left(1-\left|\rho_{n}(w)\right|^{2}\right)^{-1} .
\end{aligned}
$$

The superstar is with respect to the variable $z$.

Note that the $\rho_{n}(w)$ of this theorem and the $\lambda_{n}$ of the previous theorem are related by

$$
\lambda_{n}=\eta_{n} \rho_{n}\left(\alpha_{n-1}\right), \quad \text { with } \quad \eta_{n}=\frac{1-\bar{\alpha}_{n-1} \alpha_{n}}{1-\bar{\alpha}_{n} \alpha_{n-1}} z_{n} \bar{z}_{n-1} \in \mathbb{T} .
$$

However, the recurrence for the kernels only holds for $z, w \notin \mathbb{T}$. For $w \in \mathbb{T}$, it is clear that $\rho_{n}(w) \in \mathbb{T}$, so that $1-\left|\rho_{n}(w)\right|^{2}=0$, and the recurrence breaks down. In the sections to follow, we do need the kernels for values of $w \in \mathbb{T}$, so that the recurrence of the previous theorem is useless.

Like in the polynomial case we have a certain equivalence between the existence of a recurrence relation and the orthogonality (Favard's theorem) [BGVHN92] and 
on the other hand between the recurrence relation and the existence of a ChristoffelDarboux type formula (see [Bre90] for the real line). In our case the ChristoffelDarboux relation takes the following form.

THEOREM 5.3 (Christoffel-Darboux formula). Let $\left\{\phi_{k}\right\}_{k=0}^{n}$ with $\phi_{k} \in \mathcal{L}_{k} \backslash \mathcal{L}_{k-1}$ be an orthonormal basis for $\mathcal{L}_{n}$, then the reproducing kernel $k_{n}(z, w)$ for $\mathcal{L}_{n}$ satisfies

$$
k_{n}(z, w)=\frac{\phi_{n+1}^{*}(z) \overline{\phi_{n+1}^{*}(w)}-\phi_{n+1}(z) \overline{\phi_{n+1}(w)}}{1-\zeta_{n+1}(z) \overline{\zeta_{n+1}(w)}} .
$$

For this paper, the relation with quadrature formulas will be important. The zeros of the orthogonal rational functions (ORF) are known to be in the open unit disk $\mathbb{D}$, so they are not suitable for the construction of the quadrature formulas representing integrals over the unit circle $\mathbb{T}$. We should rather have knots which are located on $\mathbb{T}$. Such knots are provided by the zeros of para-orthogonal functions. The para-orthogonal functions are defined as

$$
Q_{n}\left(z, \tau_{n}\right)=\phi_{n}(z)+\tau_{n} \phi_{n}^{*}(z)
$$

for $\tau_{n} \in \mathbb{T}, n=0,1, \ldots$ and the zeros of these are simple and on $\mathbb{T}$. We have the following rational Szegő quadrature formula.

ThEOREM 5.4 (Rational Szegö quadrature). The zeros of the para-orthogonal rational function $Q_{n+1}\left(z, \tau_{n+1}\right)$ are all simple and on $\mathbb{T}$. Let us denote them by $\left\{\xi_{n, k}\right\}_{k=0}^{n}$. Moreover, defining $\lambda_{n k}=\left[k_{n}\left(\xi_{n k}, \xi_{n k}\right)\right]^{-1}$, with $k_{n}(z, w)$ the reproducing kernel for $\mathcal{L}_{n}$, then equality holds in

$$
\langle f, g\rangle=\sum_{k=0}^{n} \lambda_{n k} f\left(\xi_{n k}\right) g_{*}\left(\xi_{n k}\right), \quad \forall f, g \in \mathcal{L}_{n} .
$$

Conversely, if the above equality holds, then the $\xi_{n k}$ should be the zeros of the paraorthogonal function $Q_{n+1}\left(z, \tau_{n+1}\right)$ for some $\tau_{n+1} \in \mathbb{T}$ and $\lambda_{n k}=\left[k_{n}\left(\xi_{n k}, \xi_{n k}\right)\right]^{-1}$.

The $n+1$ zeros $\left\{\xi_{n k}\right\}_{k=0}^{n}$ can also be characterized as follows.

THEOREM 5.5. Let $k_{n}(z, w)$ be the reproducing kernel for $\mathcal{L}_{n}$. Define $\xi_{n 0}=w$ with $w$ arbitrary on $\mathbb{T}$ and $\left\{\xi_{n k}\right\}_{k=1}^{n}$ the $n$ zeros of $k_{n}(z, w)$. Then the numbers $\left\{\xi_{n k}\right\}_{k=0}^{n}$ are the zeros of the para-orthogonal function $Q_{n+1}\left(z, \tau_{n+1}\right)$ with

$$
\tau_{n+1}=-\frac{\phi_{n+1}(w)}{\phi_{n+1}^{*}(w)} .
$$

Conversely, if $\left\{\xi_{n k}\right\}_{k=0}^{n}$ are the zeros of some para-orthogonal function $Q_{n+1}\left(z, \tau_{n+1}\right)$, then there exists a number $w \in \mathbb{T}$, such that $\xi_{n 0}=w$ and $\tau_{n+1}=-\frac{\phi_{n+1}(w)}{\phi_{n+1}^{*}(w)}$, while $\left\{\xi_{n k}\right\}_{k=1}^{n}$ are the $n$ zeros of $k_{n}(z, w)$.

Proof. First note that neither $\phi_{n+1}$ nor $\phi_{n+1}^{*}$ can be zero on $\mathbb{T}$. Thus $\tau_{n+1}$ is well defined and is on $\mathbb{T}$ for all $w \in \mathbb{T}$ because $\left|\phi_{n+1}^{*}\right|=\left|B_{n+1}\right|\left|\phi_{n+1}\right|=\left|\phi_{n+1}\right|$ on $\mathbb{T}$. Thus it is immediately seen that the zeros of $Q_{n+1}\left(z, \tau_{n+1}\right)$ are the zeros of

$$
\phi_{n+1}^{*}(z) \overline{\phi_{n+1}^{*}(w)}-\phi_{n+1}(z) \overline{\phi_{n+1}(w)} \text {. }
$$

Obviously $z=w$ is one of the zeros. In the Christoffel-Darboux formula, this zero is canceled by the denominator and it is of course not a zero of $k_{n}(z, w)$. The $n$ other zeros of $Q_{n+1}\left(z, \tau_{n+1}\right)$ however are precisely the zeros of $k_{n}(z, w)$.

Conversely, recall that all the zeros of $\phi_{n+1}$ are in $\mathbb{D}$ and hence none of the zeros of $\phi_{n+1}^{*}$ are in $\mathbb{D} \cup \mathbb{T}$. Thus it follows that $-\phi_{n+1} / \phi_{n+1}^{*}$ has winding number 
$n+1$, so that there are $n+1$ values of $w \in \mathbb{T}$ such that $\tau_{n+1}=-\phi_{n+1}(w) / \phi_{n+1}^{*}(w)$. Each of these values of $w$ has to be a zero of $Q_{n+1}\left(z, \tau_{n+1}\right)$, thus we can pick the one which is $\xi_{n 0}$. The other zeros of $Q_{n+1}\left(z, \tau_{n+1}\right)$ have to be zeros of $k_{n}(z, w)$ as was explained above.

\section{The ORK basis}

We have discussed before why we want to replace the ORF basis by a basis formed by reproducing kernels. By Corollary 4.3 , we know that $\left\{k_{n}\left(z, x_{j}\right)\right\}_{j=0}^{n}$ forms a basis for $\mathcal{L}_{n}$ for almost any set of distinct points $\boldsymbol{x}=\left\{x_{j}\right\}$. The question is whether it is possible to choose the points in $\boldsymbol{x}$ such that this basis is orthogonal. In that case we would have a basis of orthogonal rational kernels (ORK).

It turns out that if we choose $x_{j}=\xi_{n j}, j=0, \ldots, n$ the zeros of a paraorthogonal function $Q_{n+1}\left(z, \tau_{n+1}\right)$, then the basis $\left\{\varphi_{n j}(z)=k_{n}\left(z, \xi_{n j}\right)\right\}_{j=0}^{n}$ is orthogonal.

THEOREM 6.1. Let $k_{n}(z, w)$ be the reproducing kernel for $\mathcal{L}_{n}$ and let $\boldsymbol{\xi}_{n}=$ $\left\{\xi_{n j}\right\}_{j=0}^{n}$ be the zeros of a para-orthogonal function $Q_{n+1}\left(z, \tau_{n+1}\right)$ for some $\tau_{n+1} \in$ $\mathbb{T}$. Then the basis for $\mathcal{L}_{n}$ defined by

$$
\varphi_{n j}(z)=k_{n}\left(z, \xi_{n j}\right), \quad j=0,1, \ldots, n
$$

is orthogonal.

Proof. Let $\Phi_{n}=\Phi_{n}\left(\boldsymbol{\xi}_{n}\right)$ be defined by (4.1) then it follows from (4.2) and from

$$
\left\langle\varphi_{n i}, \varphi_{n j}\right\rangle=\left\langle k_{n}\left(z, \xi_{n i}\right), k_{n}\left(z, \xi_{n j}\right)\right\rangle=k_{n}\left(\xi_{n j}, \xi_{n i}\right), \quad i, j=0, \ldots, n
$$

that $\left\{\varphi_{n j}\right\}_{j=0}^{n}$ will be an orthogonal basis if and only if

$$
k_{n}\left(\xi_{n j}, \xi_{n i}\right)=\delta_{i j} k_{n}\left(\xi_{n i}, \xi_{n i}\right), \quad i, j=0, \ldots, n .
$$

In terms of the matrix (4.1) this reads

$$
\Phi_{n}^{H} \Phi_{n}=\Lambda_{n}^{-1} \quad \text { or equivalently } \quad \Phi_{n} \Lambda_{n} \Phi_{n}^{H}=I
$$

with $\Lambda_{n}=\operatorname{diag}\left(\lambda_{n 0}, \ldots, \lambda_{n n}\right)$ a diagonal matrix with $\lambda_{n i}=1 / k_{n}\left(\xi_{n i}, \xi_{n i}\right)$. Writing $\Phi_{n} \Lambda_{n} \Phi_{n}^{H}=I$ explicitly gives

$$
\sum_{k=0}^{n} \lambda_{n k} \phi_{i}\left(\xi_{n k}\right) \overline{\phi_{j}\left(\xi_{n k}\right)}=\delta_{i j}, \quad i, j=0, \ldots, n .
$$

Because also $\left\langle\phi_{i}, \phi_{j}\right\rangle=\delta_{i j}$ for $i, j=0, \ldots, n$, this means that the above quadrature formula is exact for the inner product of all basis functions in $\mathcal{L}_{n}$, hence for the inner product of any two functions in $\mathcal{L}_{n}$. By Theorem 5.4, this means that it can only be the rational Szegö quadrature formula. Thus the theorem follows.

EXAMPLE 6.1. Let us consider again the trivial example where all $\alpha_{k}=0$ and where $\mu$ is the normalized Lebesgue measure on $\mathbb{T}$. It is clear that if we set $\tau_{n+1}=$ -1 , then $Q_{n+1}\left(z, \tau_{n+1}\right) \equiv z^{n+1}-1$. Thus its zeros are given by $\left\{\xi_{n k}=e^{-i 2 k \pi /(n+1)}\right\}$ for $k=0, \ldots, n$. Supposing that $f_{n} \in \mathcal{L}_{n}$ is given by $f_{n}(z)=\sum_{k=0}^{n} a_{n k} \phi_{k}(z)=$ $\sum_{j=0}^{n} p_{n j} k_{n}\left(z, \xi_{n j}\right)$, thus

$$
f_{n}\left(e^{i \theta}\right)=\sum_{k=0}^{n} a_{n k} e^{i k \theta}=\sum_{k=0}^{n} e^{i k \theta} \sum_{j=0}^{n} p_{n j} e^{i 2 j k \pi /(n+1)}
$$


it follows that the $\left\{a_{n k}\right\}$ are given by the Discrete Fourier Transform (DFT) of $\left\{p_{n j}\right\}$. In other words, the matrix $\Phi_{n}\left(\boldsymbol{\xi}_{n}\right)$ becomes a Fourier matrix.

\section{The WRK basis}

We now consider the problem to find a reproducing kernel basis for the spaces $\mathcal{K}_{n}=\mathcal{L}_{2 n} \ominus \mathcal{L}_{n}$. Obviously the reproducing kernel for $\mathcal{K}_{n}$ is

$$
l_{n}(z, w)=k_{2 n}(z, w)-k_{n}(z, w)=\sum_{k=n+1}^{2 n} \phi_{k}(z) \overline{\phi_{k}(w)} .
$$

The main question in this respect is: Can we find $n$ numbers $\left\{\eta_{n j}\right\}_{j=0}^{n-1}$ such that the functions $\left\{\psi_{n j}(z)=l_{n}\left(z, \eta_{n j}\right)\right\}_{j=0}^{n-1}$ form a basis for $\mathcal{K}_{n}$ and if possible, can it be made orthogonal?

We have to investigate the matrix

$$
\Psi_{n}(\boldsymbol{y})=\left[\begin{array}{ccc}
\phi_{n+1}\left(y_{0}\right) & \cdots & \phi_{n+1}\left(y_{n-1}\right) \\
\vdots & & \vdots \\
\phi_{2 n}\left(y_{0}\right) & \cdots & \phi_{2 n}\left(y_{n-1}\right)
\end{array}\right]
$$

Using the following lemma, it is possible to prove that there always exists a set of points $\left\{y_{j}\right\}_{j=0}^{n-1}$ on $\mathbb{T}$ which make this matrix regular.

Lemma 7.1. Assume that $Q \in \mathbb{C}^{(2 n+1) \times(2 n+1)}$ is a square matrix such that $Q^{H} Q=D$ with $D$ regular and diagonal. Assume moreover that this $Q$ is subdivided as

$$
Q=\left[\begin{array}{ll}
Q_{1} & Q_{2} \\
Q_{3} & Q_{4}
\end{array}\right]
$$

with $Q_{1} \in \mathbb{C}^{(n+1) \times(n+1)}$ and hence $Q_{4} \in \mathbb{C}^{n \times n}$. If $Q_{1}$ is regular and if $Q_{2} \in \mathbb{C}^{n \times n+1}$ is of full rank $n$, then $Q_{4}$ is regular.

Proof. Let $D$ be subdivided into the two parts $D_{1} \in \mathbb{R}^{(n+1) \times(n+1)}$ and $D_{2} \in$ $\mathbb{R}^{n \times n}$. We know from $Q^{H} Q=D$ that

$$
Q_{2}^{H} Q_{1}+Q_{4}^{H} Q_{3}=0
$$

so that $\operatorname{rank}\left(Q_{2}^{H} Q_{1}\right)=\operatorname{rank}\left(Q_{4}^{H} Q_{3}\right)$. Because $Q_{1}$ is regular and $\operatorname{rank}\left(Q_{2}\right)=n$, we find that $\operatorname{rank}\left(Q_{4}^{H} Q_{3}\right)=n$. Now if $Q_{4}$ were singular, then there would exist a nonzero vector $\mathbf{c} \in \mathbb{C}^{1 \times n}$ such that $\mathbf{c} Q_{4}^{H}=0$, hence also $\mathbf{c} Q_{4}^{H} Q_{3}=0$. In other words, $\operatorname{rank}\left(Q_{4}^{H} Q_{3}\right)<n$, which is a contradiction.

This makes it possible to prove the following theorem which ensures the existence of a wavelet reproducing kernel basis (WRK).

Theorem 7.2. Consider the zeros $\boldsymbol{\xi}_{2 n}=\left\{\xi_{2 n, k}: k=0,1, \ldots, 2 n\right\}$ of the paraorthogonal function $Q_{2 n+1}\left(z, \tau_{2 n+1}\right)$ for some $\tau_{2 n+1} \in \mathbb{T}$. If we select $\boldsymbol{y}_{n}=\left\{y_{k}\right.$ : $k=0, \ldots, n-1\}$ to be any $n$ out of the $2 n+1$ zeros in $\boldsymbol{\xi}_{2 n}$, then the matrix $\Psi_{n}\left(\boldsymbol{y}_{n}\right)$ as defined in (7.1) will be regular.

PRoof. Since the ordering of the zeros $\xi_{2 n, k}$ is completely arbitrary, we can always assume that we select the $y_{k}$ to be the last elements of $\boldsymbol{\xi}_{2 n}=\left\{\xi_{2 n, j}: j=\right.$ $0, \ldots, 2 n\}$. Now consider the matrix $\Phi_{2 n}=\Phi_{2 n}\left(\xi_{2 n}\right)$ of (4.1) where the evaluation is in the points of $\boldsymbol{\xi}_{2 n}$, then $\Psi_{n}\left(\boldsymbol{y}_{n}\right)$ appears as the $n \times n$ right lower part of the matrix $\Phi_{2 n}$. By Lemma 4.2, it follows that taking the block of the first $n+1$ 
columns in $\Phi_{2 n}$, any selection of $k$ different rows from it will result in a matrix of rank $\min \{k, n+1\}$. Thus the conditions of the previous lemma are satisfied and the theorem follows.

This theorem settles the question of the existence of a WRK basis of the form $\left\{l_{n}\left(z, y_{k}\right)\right\}_{k=0}^{n-1}$. It is however not clear how to choose these $\left\{y_{k}\right\}_{k=0}^{n-1}$ to make the WRK basis orthogonal, if it is possible at all. The same problem was encountered for the polynomial case on an interval in [FP97] where an orthogonal WRK basis could only be constructed for a Chebyshev weight of the second kind.

EXAMPLE 7.1. For some particular cases, it is however possible to construct such an orthogonal WRK basis. We give a trivial example. Consider again the case where all $\alpha_{k}=0$ and where orthogonality is with respect to the normalized Lebesgue measure of $\mathbb{T}$. In that case

$$
l_{n}(z, w)=\sum_{k=n+1}^{2 n}(z \bar{w})^{k} .
$$

Note that $\mathcal{L}_{n}=\Pi_{n}$ and $\mathcal{K}_{n}=\mathcal{L}_{2 n} \ominus \mathcal{L}_{n}=z^{n+1} \Pi_{n-1}$. Furthermore, for some distinct $\eta_{n k}, k=0, \ldots, n-1$, all on $\mathbb{T}$ define the functions $\psi_{n k}(z)=l_{n}\left(z, \eta_{n k}\right)$, $k=0, \ldots, n-1$. In analogy with Theorem 6.1 , for these to be an orthogonal basis of $\mathcal{K}_{n}$, we need the existence of numbers $\lambda_{n k}, k=0, \ldots, n-1$ such that

$$
\sum_{k=0}^{n-1} \lambda_{n k} \phi_{i}\left(\eta_{n k}\right) \overline{\phi_{j}\left(\eta_{n k}\right)}=\delta_{i j}, \quad i, j=n+1, \ldots, 2 n .
$$

In other words, we need a rational Szegö quadrature formula which is exact in $\mathcal{K}_{n}=z^{n+1} \Pi_{n-1}$. Because $f(z)=z^{n+1} p_{n-1}(z)$ and $g(z)=z^{n+1} q_{n-1}(z)$ are both in $\mathcal{K}_{n}$ if $p_{n-1}, q_{n-1} \in \Pi_{n-1}$, we have

$$
\langle f, g\rangle=\left\langle p_{n-1}, q_{n-1}\right\rangle .
$$

The quadrature formula will be exact if and only if the nodes $\eta_{n k}$ are the zeros of the para-orthogonal polynomial $Q_{n}(z, \tau)=z^{n}+\tau, \tau \in \mathbb{T}$ and the weights are given by

$$
\lambda_{n k}=\frac{1}{k_{n-1}\left(\eta_{n k}, \eta_{n k}\right)}=\frac{1}{\sum_{k=0}^{n-1}\left|\eta_{n k}\right|^{2 k}}=\frac{1}{n} .
$$

Thus in this case, one can take for example the $n$th roots of unity for $\eta_{n k}$ and the basis $\psi_{n k}$ will be an orthogonal WRK basis. Note that here also the matrix $\Psi_{n}\left(\boldsymbol{\eta}_{n}\right)$ will be the matrix of a DFT.

We can push this example a bit further in two directions. First by considering orthogonal polynomials with respect to a rational modification of the Lebesgue measure. A rational modification of the Lebesgue measure is defined as a measure $\mu$ satisfying for some $a_{j} \in \mathbb{D}, j=1, \ldots, m$

$$
\mathrm{d} \mu(t)=\frac{\mathrm{d} \lambda(t)}{|h(t)|^{2}}, \quad h(t)=\prod_{j=1}^{m}\left(1-\bar{a}_{j} t\right), \quad t=e^{i \theta}
$$

where $\lambda$ is the normalized Lebesgue measure. Orthogonal polynomials for such a measure and corresponding quadrature formulas were considered in [GVSLN96], see also [Sze67, p.289-290]. The orthogonal polynomials for such a measure are given by $\phi_{k}(z)=z^{k-m} h^{*}(z)=z^{k} h_{*}(z)$ for $k \geq m$ (see [Sze67, Thm. 11.2, p.289]). 
For the polynomial $h(z)$ of degree $m$ we have used $h^{*}(z)=z^{m} h_{*}(z)=\prod_{j=1}^{m}\left(z-a_{j}\right)$. Thus for $n+1 \geq m$, it follows that $\mathcal{K}_{n}=\operatorname{span}\left\{\phi_{n+1}, \ldots, \phi_{2 n}\right\}=z^{n+1-m} h^{*}(z) \Pi_{n-1}$. Therefore, if $f, g \in \mathcal{K}_{n}$, then they can be represented as $f(z)=z^{n+1-m} h^{*}(z) p_{n-1}(z)$ and $g(z)=z^{n+1-m} h^{*}(z) q_{n-1}(z)$ with $p_{n-1}, q_{n-1} \in \Pi_{n-1}$ and we have $\langle f, g\rangle_{\mu}=$ $\left\langle p_{n-1}, q_{n-1}\right\rangle_{\lambda}$.

If we want $\left\{\psi_{n k}(z)=l_{n}\left(z, \eta_{n k}\right)\right\}$ to be an orthogonal WRK basis, then, as shown before, the numbers $\eta_{n k}$ should be chosen such that there exist positive numbers $\lambda_{n k}$ such that (7.2) holds, hence such that

$$
\sum_{k=0}^{n-1} \lambda_{n k} \eta_{n k}^{i-j}\left|h^{*}\left(\eta_{n k}\right)\right|^{2}=\left\langle\phi_{i}, \phi_{j}\right\rangle_{\mu}=\left\langle z^{i-n-1}, z^{j-n-1}\right\rangle_{\lambda} .
$$

This is clearly possible by choosing $\eta_{n k}$ to be the $n$ zeros of the para-orthogonal polynomial $z^{n}+\tau, \tau \in \mathbb{T}$ (and the weights are $\left.\lambda_{n k}=\left|h\left(\eta_{n k}\right)\right|^{2} / n\right)$.

A second generalization of the simple case that was discussed above is to consider orthogonal rational functions with respect to the Lebesgue measure. Such a situation was considered in [BGVHN94]. In this case the orthogonal rational functions are given by

$$
\phi_{k}(z)=\sqrt{1-\left|\alpha_{k}\right|^{2}} \frac{z B_{k}(z)}{z-\alpha_{k}}
$$

Therefore $\mathcal{K}_{n}=\mathcal{L}_{2 n} \ominus \mathcal{L}_{n}=\operatorname{span}\left\{\phi_{n+1}, \ldots, \phi_{2 n}\right\}$ is given by

$$
\mathcal{K}_{n}=\beta_{n+1} \tilde{\mathcal{L}}_{n-1}, \quad \beta_{n+1}(z)=\frac{z B_{n+1}(z)}{1-\bar{\alpha}_{n+1} z}
$$

where $\tilde{\mathcal{L}}_{n-1}$ is the space of rational functions associated with the points $\left\{\tilde{\alpha}_{j}=\right.$ $\left.\alpha_{n+1+j}\right\}_{j=1}^{n-1}$. Thus, if $f, g \in \mathcal{K}_{n}$, then there exist $\tilde{p}_{n-1}, \tilde{q}_{n-1} \in \tilde{\mathcal{L}}_{n-1}$ such that

$$
f(z)=\beta_{n+1}(z) \tilde{p}_{n-1}(z), \quad g(z)=\beta_{n+1}(z) \tilde{q}_{n-1}(z),
$$

and therefore

$$
\langle f, g\rangle_{\lambda}=\int_{\mathbb{T}} f(t) \overline{g(t)} \mathrm{d} \lambda(t)=\int_{\mathbb{T}} \tilde{p}_{n-1} \overline{\tilde{q}_{n-1}(t)} \mathrm{d} \mu(t)=\left\langle\tilde{p}_{n-1}, \tilde{q}_{n-1}\right\rangle_{\mu}
$$

with $\mathrm{d} \mu(t)=\left|t-\alpha_{n+1}\right|^{-2} \mathrm{~d} \lambda(t)$. Note that this is a rational modification of the Lebesgue measure which is, up to the factor $1-\left|\alpha_{n+1}\right|^{2}$ equal to the Poisson kernel $P\left(t, \alpha_{n+1}\right)=\left(1-\left|\alpha_{n+1}\right|^{2}\right) /\left|t-\alpha_{n+1}\right|^{2}$. In [BGVHN94, Section 3] weights and abscisses were given for this case which give an $n$-point rational Szegő quadrature formula which is exact in $\tilde{\mathcal{L}}_{(n-1) *} \cdot \tilde{\mathcal{L}}_{n-1}$, hence for which the inner product $(7.3)$ is evaluated exactly. Thus also in this case we can construct an orthogonal wavelet basis $\left\{\psi_{n k}(z)=l_{n}\left(z, \eta_{n k}\right)\right\}_{k=0}^{n-1}$.

EXAMPLE 7.2. Let us give a less trivial example. We choose equispaced $\alpha_{k}$ on the circle $|z|=0.9$ as shown in Figure 2. The reproducing kernels for the Lebesgue measure on the unit circle are computed and their real, and imaginary part, and their modulus are plotted, both for the kernels $k_{n}$ and for the kernels $l_{n}$. The result is shown in Figure 2. 
FiguRE 2. The real and imaginary part and the modulus of the functions $k_{8}(z, w)$ (top) and $l_{8}(z, w)$ (bottom) for $z=e^{i \theta}$ and $w=$ 1 , plotted as functions of $\theta$.The measure is the normalized Lebesgue measure and the $\alpha_{k}$ are chosen as $\alpha_{k}=0.9 e^{i 2 k \pi / 8}$ for $k=1, \ldots, 8$ and $\alpha_{k+8}=0.9 e^{i(2 k+1) \pi / 8}$ for $k=1, \ldots, 8$. These $\alpha_{k}$ 's are plotted in the figure on the right.
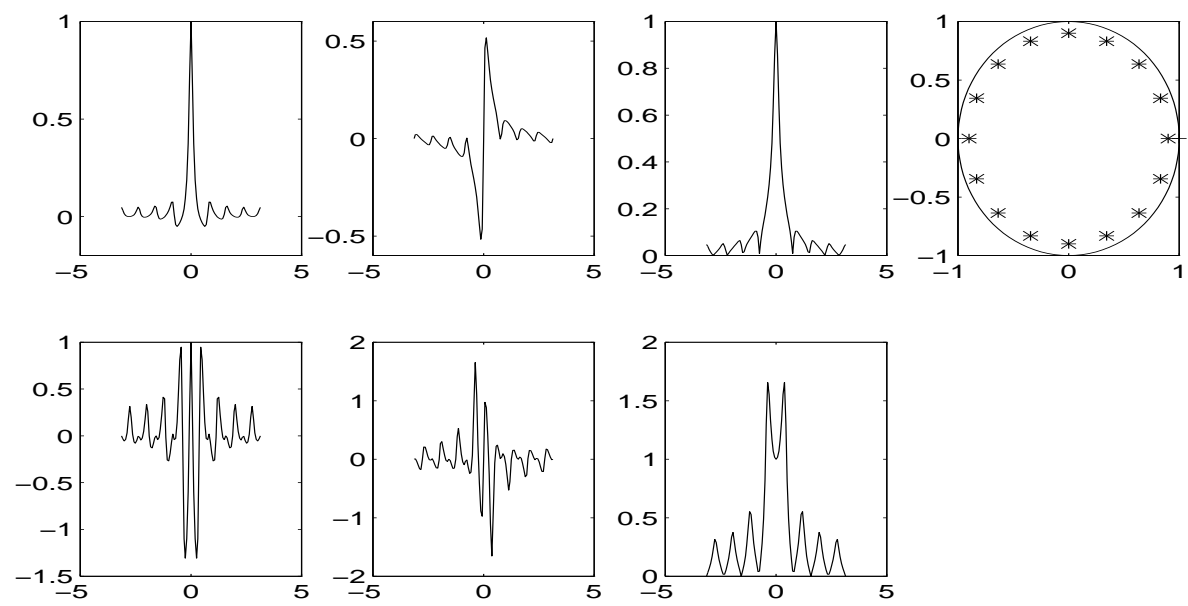

\section{Biorthogonal basis functions and interpolation}

For several reasons, it is important to have a biorthogonal basis in wavelet theory. Like in the polynomial case, the biorthogonal basis functions for the reproducing kernel bases are given by interpolation functions. This is what we shall explain in this section.

Let us define the Lagrange polynomials in $\Pi_{n}$ for the interpolation points $\boldsymbol{x}_{n}=$ $\left\{x_{k}\right\}_{k=0}^{n}($ all distinct and on $\mathbb{T})$ by

and define

$$
l_{n k}(z)=\frac{\prod_{j=0, j \neq k}^{n}\left(z-x_{j}\right)}{\prod_{j=0, j \neq k}^{n}\left(x_{k}-x_{j}\right)}, \quad k=0, \ldots, n
$$

$$
L_{n k}(z)=l_{n k}(z) \frac{\pi_{n}\left(x_{k}\right)}{\pi_{n}(z)}, \quad k=0, \ldots, n
$$

with as before $\pi_{n}(z)=\prod_{j=0}^{n}\left(1-\bar{\alpha}_{j} z\right)$. Then obviously $L_{n k}\left(x_{j}\right)=\delta_{k j}, k, j=$ $0, \ldots, n$ while $L_{n k} \in \mathcal{L}_{n}$. We call these $L_{n k}$ the fundamental Lagrange interpolating functions (FLIF) of $\mathcal{L}_{n}$ for the points $\boldsymbol{x}_{n}=\left\{x_{k}\right\}_{k=0}^{n}$. It immediately follows that for any function $f \in \mathcal{L}_{n}$ we may write

$$
f(z)=\sum_{k=0}^{n} f\left(x_{k}\right) L_{n k}(z)
$$

Defining in $\mathcal{L}_{n}$ the discrete inner product

$$
\langle f, g\rangle_{\boldsymbol{x}_{n}}=\sum_{k=0}^{n} f\left(x_{k}\right) \overline{g\left(x_{k}\right)}
$$


it is directly seen that the FLIF $\left\{L_{n k}\right\}_{k=0}^{n}$ form an orthonormal basis for $\mathcal{L}_{n}$ with respect to this inner product. Note that the reproducing kernels for $\mathcal{L}_{n}$ with respect to this inner product are given by $\sum_{j=0}^{n} L_{n j}(z) \overline{L_{n j}\left(x_{k}\right)}=L_{n k}(z)$. Thus the $L_{n k}$ are reproducing kernels and by the general theory we may say that among all functions in $\mathcal{L}_{n}$ that take the value 1 at $x_{k}$, they will have minimal norm $\left\|L_{n k}\right\|_{\boldsymbol{x}_{n}}$.

If we set $\varphi_{n k}(z)=k_{n}\left(z, x_{k}\right)$ and $\tilde{\varphi}_{n k}(z)=L_{n k}(z)$, then because of the reproducing property of $\varphi_{n k}$

$$
\left\langle\tilde{\varphi}_{n k}, \varphi_{n j}\right\rangle=\delta_{k j} .
$$

In other words, $\varphi_{n k}$ and $\tilde{\varphi}_{n k}$ are biorthogonal bases for $\mathcal{L}_{n}$.

Note that we can characterize $\tilde{\varphi}_{n k}$ in another way. We may write $\tilde{\varphi}_{n k}=$ $\sum_{j=0}^{n} c_{j}^{(k)} \phi_{j}$ where $\boldsymbol{c}^{(k)}=\left[c_{0}^{(k)} \ldots c_{n}^{(k)}\right]$ is defined by $\boldsymbol{c}^{(k)} \Phi_{n}=\boldsymbol{e}_{k}$ where $\boldsymbol{e}_{k}=$


$c^{(k)}$ is row $k$ in the inverse of $\Phi_{n}$, so that we have

$$
\left[\begin{array}{c}
\tilde{\varphi}_{n 0} \\
\vdots \\
\tilde{\varphi}_{n n}
\end{array}\right]=\left[\begin{array}{ccc}
c_{0}^{(0)} & \cdots & c_{n}^{(0)} \\
\vdots & & \vdots \\
c_{0}^{(n)} & \cdots & c_{n}^{(n)}
\end{array}\right]\left[\begin{array}{c}
\phi_{0} \\
\vdots \\
\phi_{n}
\end{array}\right]=\Phi_{n}^{-1}\left[\begin{array}{c}
\phi_{0} \\
\vdots \\
\phi_{n}
\end{array}\right]
$$

It is clear that if the $x_{k}$ are the zeros of a para-orthogonal polynomial $Q_{n+1}\left(z, \tau_{n+1}\right)$, then the $\varphi_{n k}$ are orthogonal and $\left\langle\varphi_{n i}, \varphi_{n j}\right\rangle=\delta_{i j} \varphi_{n i}\left(x_{i}\right)$. In that case we have of course $L_{n k}(z)=\varphi_{n k}(z) / \varphi_{n k}\left(x_{k}\right)$.

Similarly, given $\boldsymbol{y}_{n}=\left\{y_{k}\right\}_{k=0}^{n-1}$, one can construct a biorthogonal basis for $\psi_{n k}(z)=l_{n}\left(z, y_{k}\right)$, on condition that the matrix $\Psi_{n}=\Psi_{n}\left(\boldsymbol{y}_{n}\right)$ of (7.1) is regular. Indeed, let $\boldsymbol{e}_{k}=\left[\begin{array}{lllll}0 & \ldots & 0 & 1 & 0\end{array} \ldots .0\right]$ be the $k$ th unit vector, then if $\Psi_{n}$ is regular, there is exactly one solution $\boldsymbol{d}^{(k)}=\left[d_{n+1}^{(k)} \ldots d_{2 n}^{(k)}\right]$ to the equation $\boldsymbol{d}^{(k)} \Psi_{n}=\boldsymbol{e}_{k}$, for each $k=0, \ldots, n-1$. The function $\tilde{\psi}_{n k}(z)=\sum_{j=n+1}^{2 n} d_{j}^{(k)} \psi_{n j}(z)$ is obviously in $\mathcal{K}_{n}$ and we have $\tilde{\psi}_{n k}\left(y_{j}\right)=\delta_{k j}, k, j=0, \ldots, n-1$. Thus these $\tilde{\psi}_{n k}$ form the FLIF of $\mathcal{K}_{n}$ for the interpolation points $\boldsymbol{y}_{n}=\left\{y_{k}\right\}_{k=0}^{n-1}$. We can write for any function $f \in \mathcal{K}_{n}$ that

$$
f(z)=\sum_{k=0}^{n-1} f\left(y_{k}\right) \tilde{\psi}_{n k}(z) .
$$

The $\tilde{\psi}_{n k}$ are orthonormal with respect to the discrete inner product

$$
\langle f, g\rangle_{\boldsymbol{y}_{n}}=\sum_{k=0}^{n-1} f\left(y_{k}\right) \overline{g\left(y_{k}\right)}
$$

and $\tilde{\psi}_{n k}$ is the solution to the problem

$$
\min \left\{\|f\|_{\boldsymbol{y}_{n}}^{2}: f\left(y_{k}\right)=1 ; f \in \mathcal{K}_{n}\right\} .
$$

Moreover, they form a biorthogonal basis for the $\psi_{n k}$ in $\mathcal{K}_{n}$ because by the reproducing property of $\psi_{n k}$ we have $\left\langle\tilde{\psi}_{n k}, \psi_{n j}\right\rangle=\delta_{k j}, k, j=0, \ldots, n-1$. The relation between the bases $\left\{\phi_{k}\right\}_{k=n+1}^{2 n}$ and $\left\{\tilde{\psi}_{n k}\right\}_{k=0}^{n-1}$ is given by

$$
\left[\begin{array}{c}
\tilde{\psi}_{n 0} \\
\vdots \\
\tilde{\psi}_{n, n-1}
\end{array}\right]=\left[\begin{array}{ccc}
d_{n+1}^{(0)} & \cdots & d_{2 n}^{(0)} \\
\vdots & & \vdots \\
d_{n+1}^{(n-1)} & \cdots & d_{2 n}^{(n-1)}
\end{array}\right]\left[\begin{array}{c}
\phi_{n+1} \\
\vdots \\
\phi_{2 n}
\end{array}\right]=\Psi_{n}^{-1}\left[\begin{array}{c}
\phi_{n+1} \\
\vdots \\
\phi_{2 n}
\end{array}\right]
$$


with $\Psi_{n}=\Psi_{n}\left(\boldsymbol{y}_{n}\right)$.

\section{Decomposition and reconstruction formulas}

We are now in a position to give concrete formulas for the decomposition and reconstruction steps that were described in Section 3.

Assume that for each $n$, we select two sets of distinct points $\boldsymbol{x}_{n}=\left\{x_{n k}\right\}_{k=0}^{n}$ and $\boldsymbol{y}_{n}=\left\{y_{n k}\right\}_{k=0}^{n-1}$ on $\mathbb{T}$ with $\boldsymbol{y}_{n}$ such that $\Psi_{n}\left(\boldsymbol{y}_{n}\right)$ of (7.1) is regular. We write in short hand $\Phi_{n}=\Phi_{n}\left(\boldsymbol{x}_{n}\right)$ and $\Psi_{n}=\Psi_{n}\left(\boldsymbol{y}_{n}\right)$. In that case $\varphi_{n k}(z)=k_{n}\left(z, x_{n k}\right)$ forms a basis for $\mathcal{L}_{n}$ and $\psi_{n k}(z)=k_{2 n}\left(z, y_{n k}\right)-k_{n}\left(z, y_{n k}\right)$ is a basis for $\mathcal{K}_{n}$ when $k_{n}(z, w)$ is the reproducing kernel for $\mathcal{L}_{n}$. We use the notation of Section 3 and set for $f_{2 n} \in \mathcal{L}_{2 n} f_{2 n}=\boldsymbol{p}_{2 n} \boldsymbol{\varphi}_{2 n}=f_{n}+g_{n}$ with $f_{n}=\boldsymbol{p}_{n} \boldsymbol{\varphi}_{n} \in \mathcal{L}_{n}$ and $g_{n}=\boldsymbol{q}_{n} \boldsymbol{\psi}_{n} \in \mathcal{K}_{n}$. On the other hand, setting $\phi_{n}=\left[\phi_{0}, \phi_{1}, \ldots, \phi_{n}\right]^{T}$ and $\boldsymbol{\phi}_{n}^{c}=\left[\phi_{n+1}, \phi_{n+2}, \ldots, \phi_{2 n}\right]^{T}$, then $\boldsymbol{\varphi}_{n}=\Phi_{n}^{H} \phi_{n}$ and $\boldsymbol{\psi}_{n}=\Psi_{n}^{H} \phi_{n}^{c}$. Hence

$$
f_{2 n}=\boldsymbol{p}_{2 n} \Phi_{2 n}^{H} \boldsymbol{\phi}_{2 n}, f_{n}=\boldsymbol{p}_{n} \Phi_{n}^{H} \boldsymbol{\phi}_{n}, g_{n}=\boldsymbol{q}_{n} \Psi_{n}^{H} \boldsymbol{\phi}_{n}^{c} .
$$

Equating coefficients of the corresponding basis functions in $f_{2 n}=f_{n}+g_{n}$ leads to

$$
\boldsymbol{p}_{2 n} \Phi_{2 n}^{H}=\left[\boldsymbol{p}_{n} \boldsymbol{q}_{n}\right]\left[\begin{array}{cc}
\Phi_{n}^{H} & 0 \\
0 & \Psi_{n}^{H}
\end{array}\right] .
$$

This relation allows us to compute $\boldsymbol{p}_{n}$ and $\boldsymbol{q}_{n}$ from $\boldsymbol{p}_{2 n}$ and conversely to reconstruct $\boldsymbol{p}_{2 n}$ from $\boldsymbol{p}_{n}$ and $\boldsymbol{q}_{n}$.

For example, using the relations $p_{n r}=\left\langle f_{2 n}, \tilde{\varphi}_{n r}\right\rangle$ and $q_{n r}=\left\langle f_{2 n}, \tilde{\psi}_{n r}\right\rangle$ we find that

$$
p_{n r}=\sum_{k=0}^{2 n} p_{2 n, k} \overline{\tilde{\varphi}_{n r}\left(x_{2 n, k}\right)}, \quad r=0, \ldots, n .
$$

and

$$
q_{n r}=\sum_{k=0}^{2 n} p_{2 n, k} \overline{\tilde{\psi}_{n r}\left(x_{2 n, k}\right)}, \quad r=0, \ldots, n-1 .
$$

In the special case where the $\varphi_{n, k}$ are orthogonal, i.e., when the $x_{n, k}$ are the zeros $\xi_{n, k}$ of $Q_{n+1}\left(z, \tau_{n+1}\right)$, then $\tilde{\varphi}_{n, r}=\varphi_{n, r} / \varphi_{n, r}\left(\xi_{n, r}\right)$ and we get for the first of the analysis formulas

$$
p_{n r}=\frac{1}{\varphi_{n, r}\left(\xi_{n, r}\right)} \sum_{k=0}^{2 n} p_{2 n, k} \overline{\varphi_{n r}\left(x_{2 n, k}\right)}, \quad r=0, \ldots, n .
$$

In general, the $\psi_{n k}$ are not orthogonal, but if we choose $\left\{y_{n r}\right\}_{r=0}^{n-1}$ to be the last $n$ zeros $\left\{\xi_{2 n, n+1+r}\right\}_{r=0}^{n-1}$, then by definition $\tilde{\psi}_{n r}\left(y_{n s}\right)=\delta_{r s}, r, s=0, \ldots, n-1$. Thus, the second analysis formula reduces to

$$
q_{n r}=\sum_{k=0}^{n} p_{2 n, k} \overline{\tilde{\psi}_{n r}\left(\xi_{2 n, k}\right)}+p_{2 n, n+1+r}, \quad r=0, \ldots, n-1 .
$$

For the reconstruction formulas, it follows from $p_{2 n, r}=\left\langle f_{n}+g_{n}, \tilde{\varphi}_{2 n, r}\right\rangle$ that

$$
p_{2 n, r}=\sum_{k=0}^{n} p_{n k}\left\langle\varphi_{n k}, \tilde{\varphi}_{2 n, r}\right\rangle+\sum_{l=0}^{n-1} q_{n l}\left\langle\psi_{n l}, \tilde{\varphi}_{2 n, r}\right\rangle, \quad r=0, \ldots, 2 n .
$$


Again, if the $\varphi_{2 n, r}$ are orthogonal, i.e., when the $x_{2 n, k}$ are the zeros $\xi_{2 n, k}$ of $Q_{2 n+1}\left(z, \tau_{2 n+1}\right)$, then $\tilde{\varphi}_{2 n, r}=\varphi_{2 n, r} / \varphi_{2 n, r}\left(\xi_{2 n, r}\right)$, and the reconstruction formula simplifies to

$$
p_{2 n, r}=\frac{1}{\varphi_{2 n, r}\left(\xi_{2 n, r}\right)}\left(\sum_{k=0}^{n} p_{n k} \varphi_{n k}\left(\xi_{2 n, r}\right)+\sum_{l=0}^{n-1} q_{n l} \psi_{n l}\left(\xi_{2 n, r}\right)\right), \quad r=0, \ldots, 2 n .
$$

\section{Multiresolution analysis}

Thus far we have only worked with finite dimensional spaces $\mathcal{L}_{n}$. With our notation $\mathcal{V}_{-1}=\mathcal{L}_{0}$ and $\mathcal{V}_{s}=\mathcal{L}_{n}$ where $n=2^{s}$, we have the nesting

$$
\mathcal{V}_{-1} \subset \mathcal{V}_{0} \subset \mathcal{V}_{1} \subset \cdots \subset \mathcal{V}_{s} \subset \mathcal{V}_{s+1} \subset \cdots
$$

One may wonder what space is ultimately covered when $s \rightarrow \infty$. If $\mathcal{K}$ is a subspace of the Hilbert space $\mathcal{H}$, then for the $\left\{\mathcal{V}_{k}\right\}$ to form a MRA of $\mathcal{K}$, one should have that

$$
\operatorname{clos} \mathcal{H}\left(\bigcup_{s=-1}^{\infty} \mathcal{V}_{s}\right)=\mathcal{K}
$$

Thus for our rational spaces, we should verify in which spaces the system $\left\{B_{k}\right\}_{k=0}^{\infty}$ is complete. There are several possibilities.

THEOREM 10.1. ([Ach56, p.244-246], [BGVHN98b, Corollary 7.2.4])

If $\sum_{k=1}^{\infty}\left(1-\left|\alpha_{k}\right|\right)=\infty$ then the system $\left\{B_{k}\right\}_{k=0}^{\infty}$ is complete in $H_{2}(\mathbb{T})$ (with respect to the Lebesgue measure) as well as in $H_{2}(\mathbb{T}, \mu)$.

If $\int \log \mu^{\prime}(t) \mathrm{d} \theta=-\infty\left(t=e^{i \theta}\right)$ and $\sum_{k=1}^{\infty}\left(1-\left|\alpha_{k}\right|\right)=\infty$ then the system $\left\{B_{k}\right\}_{k=0}^{\infty}$ is complete in $L_{2}(\mathbb{T}, \mu)$.

$\int \log \mu^{\prime}(t) \mathrm{d} \theta>-\infty$ is known as Szegö's condition while $\sum_{k=1}^{\infty}\left(1-\left|\alpha_{k}\right|\right)=\infty$ means that the Blaschke product $B(z)=\prod_{k=1}^{\infty} \zeta_{k}(z)$ diverges to zero. Thus the $\alpha_{k}$ should not approach the boundary $\mathbb{T}$ too fast. $H_{2}$ represents the Hardy space of functions in $L_{2}$ which are analytic in $\mathbb{D}$.

In classical multiresolution analysis (MRA) of $L_{2}$, several other conditions have to be satisfied. Some of these were recently generalized to what is called a second generation MRA by Sweldens [Swe98]. We shall show however that with a generalization of the definition of the shift operator as it was done for the polynomial case in [FP97], it is possible to stay close to the definition of a classical MRA.

First of all we use the term Fourier transform with respect to the ORF basis. Thus, if $f$ is in our function space, then we set $\mathcal{F}(f)=\left\{f_{n}^{\wedge}\right\}_{k=0}^{\infty}$ with $f_{k}^{\wedge}=\left\langle f, \phi_{k}\right\rangle$.

The scaling property of a classical MRA can be interpreted in this general setting by noting that $f \in \mathcal{V}_{s} \Leftrightarrow \operatorname{supp} \mathcal{F}(f)=\left\{0,1, \ldots, n=2^{s}\right\}$, whereas moving from $\mathcal{V}_{s}$ to $\mathcal{V}_{s+1}$ practically doubles the support of the Fourier transform.

For the shift invariance property, one should show that the basis functions of the resolution and the wavelet spaces are generated by shifts of one father function and one mother function. To obtain this property, we need an appropriate notion of a shift. To define such a shift, we fix some $\tau_{0}$ as a reference point on $\mathbb{T}$ and define a shift over $\tau$ as the effect of the shift operator $S_{\tau}$ via

$$
g=S_{\tau} f \quad \Leftrightarrow \quad \mathcal{F}(g)=\mathcal{F}(f) D_{\tau}
$$


where $D_{\tau}$ is a diagonal matrix

In other words

$$
D_{\tau}=\operatorname{diag}\left(\frac{\phi_{0}(\tau)}{\phi_{0}\left(\tau_{0}\right)}, \ldots, \frac{\phi_{n}(\tau)}{\phi_{n}\left(\tau_{0}\right)}, \ldots\right)^{H} .
$$

$$
\left(S_{\tau} f\right)_{k}^{\wedge}=f_{k}^{\wedge} \cdot \overline{\phi_{k}(\tau)} / \overline{\phi_{k}\left(\tau_{0}\right)}, \quad k=0,1, \ldots
$$

This definition can be justified as follows. Let us write $F(\theta)$ for $f\left(e^{i \theta}\right)$. Then $F(\theta)$ is a $2 \pi$-periodic function. In classical MRA for $2 \pi$ periodic functions, the orthogonal basis is the Fourier basis $\phi_{k}(t)=e^{i k t}$, a shift $F(\theta-\tau)$ has the effect that the $k$ th Fourier coefficient of $F$ is multiplied with $\overline{\phi_{k}(\tau) / \phi_{k}(0)}=e^{-i k \tau}$. Translating this to the functions $f$ defined on $\mathbb{T}$ with orthogonal basis $\phi_{k}(z)=z^{k}$, then a shift has the effect that the $k$ th Fourier coefficient is multiplied with $\overline{\phi_{k}(\xi) / \phi_{k}(1)}=\xi^{-k}$ where $\xi=e^{i \tau}$. So this is just a special case of our definition.

It is now clear that if we define

$$
\varphi_{n}(z)=\sum_{k=0}^{n} \overline{\phi_{k}\left(\tau_{0}\right)} \phi_{k}(z)=k_{n}\left(z, \tau_{0}\right),
$$

then $\varphi_{n k}(z)=k_{n}\left(z, x_{n k}\right)=S_{x_{n k}} \varphi_{n}(z)$. Similarly $\psi_{n k}=S_{y_{n k}} \psi_{n}$ where

$$
\psi_{n}(z)=\sum_{k=n+1}^{2 n} \overline{\phi_{k}\left(\tau_{0}\right)} \phi_{k}(z)=l_{n}\left(z, \tau_{0}\right) \text {. }
$$

Thus we have one father and one mother function per resolution level and the other basis functions are generalized shifts of these. Consequently, these spaces are shift-invariant, i.e., $f \in \mathcal{V}_{s} \Rightarrow S_{\tau} f \in \mathcal{V}_{s}$.

The bases used also have a Riesz property, i.e. there are constants $A \neq 0$ and $B$ such that

$$
A\left\|\boldsymbol{p}_{n}\right\| \leq\|f\| \leq B\left\|\boldsymbol{p}_{n}\right\|
$$

where the norms are 2-norms and $f \in \mathcal{V}_{s}$ is given by $f=\boldsymbol{p}_{n} \boldsymbol{\varphi}_{n}$. It follows from (4.2) and Parseval's equality that

$$
\|f\|^{2}=\left\|\boldsymbol{p}_{n} \Phi_{n}^{H} \boldsymbol{\phi}_{n}\right\|^{2}=\left\|\boldsymbol{p}_{n} \Phi_{n}^{H}\right\|^{2}
$$

and this implies that

$$
\frac{1}{\left\|\Phi_{n}^{-1}\right\|}\left\|\boldsymbol{p}_{n}\right\| \leq\|f\| \leq\left\|\Phi_{n}\right\|\left\|\boldsymbol{p}_{n}\right\| .
$$

Similarly, it holds that

$$
\frac{1}{\left\|\Psi_{n}^{-1}\right\|}\left\|\boldsymbol{q}_{n}\right\| \leq\|f\| \leq\left\|\Psi_{n}\right\|\left\|\boldsymbol{q}_{n}\right\|
$$

when $f \in \mathcal{W}_{s}$ with $f=\boldsymbol{q}_{n} \boldsymbol{\psi}_{n}$.

This generalized shift operator implies that the functions in the ORK basis look like (cyclic) shifts of each other. The same holds for the functions in the WRK basis. We give some examples.

EXAMPLE 10.1. The cyclic shift is illustrated in Figure 3. Which is again our trivial example where $\alpha_{k}=0$ for all $k$ and the measure is the normalized Lebesgue measure. If one introduces poles which are close to the unit circle $\mathbb{T}$, then this will have an influence on the form of the shifted ORK and WRK functions. For example in Figure 4, $\alpha_{1}=0.9$ while all other $\alpha_{k}=0$. This gives a pole near $z=1$, 
FIGURE 3. The real and imaginary part of the functions $k_{n}(z, w)=$ $\sum_{k=0}^{n}(z / w)^{k}$ (top) and $l_{n}(z, w)=\sum_{k=n+1}^{2 n}(z / w)^{k}$ (bottom) for $n=16$. All $\alpha_{k}=0$. In Figure A, we took $w=1$, and in Figure B, we took $w=\exp (i 4 \pi / 17)$.

$\mathrm{A}: k_{16}(z, 1)$ and $l_{16}(z, 1)$
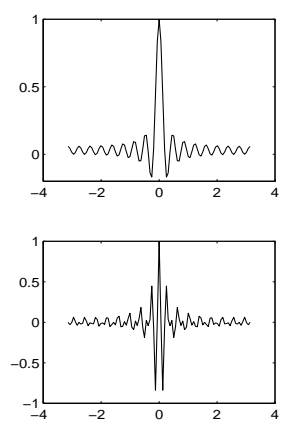
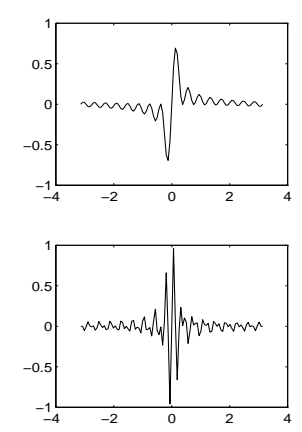

B: A "shifted" version of A


FIGURE 4. The real and imaginary part of the functions $k_{n}(z, w)$ (top) and $l_{n}(z, w)$ (bottom) for $n=16$. Here $\alpha_{1}=0.9$ while all other $\alpha_{k}=0$. In Figure A, we took $w=1$, and in Figure B, we took one of its shifts: $w=e^{i 4 \pi / 17}$.

$\mathrm{A}: k_{16}(z, 1)$ and $l_{16}(z, 1)$



B: A "shifted" version of A


i.e., near $\theta=0$. This pole forces the peak near $\theta=0$ to be more pronounced, but it also implies oscillations of the ORK and WRK functions in the neighborhood of that pole (here near $\theta=0$ ). These oscillations disappear for $w=1$ in $k_{n}(z, w)$ and $l_{n}(z, w)$, but they do show when $w$ moves away from the pole. This is almost not visible in Figure 4. However this oscillation effect increases when the pole is given more weight by making it a multiple pole. This implies a visual distortion of the simple shift-like property as in Figure 5. where we took all $\alpha_{k}=0.9, k \geq 1$. The peak at $\theta=0$ is now more pronounced than in Figure 4, and the shifted versions are heavily perturbed and do not look much like being shifted now. 
FIGURE 5. The real and imaginary part of the functions $k_{n}(z, w)$ (top) and $l_{n}(z, w)$ (bottom) for $n=16$. Here $\alpha_{k}=0.9$ for $k \geq 1$. In Figure A, we took $w=1$, and in Figure B, we took one of its shifts: $w=e^{i 4 \pi / 17}$.

$\mathrm{A}: k_{16}(z, 1)$ and $l_{16}(z, 1)$
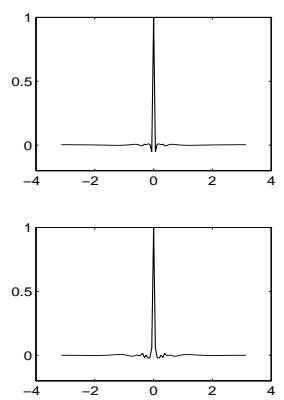
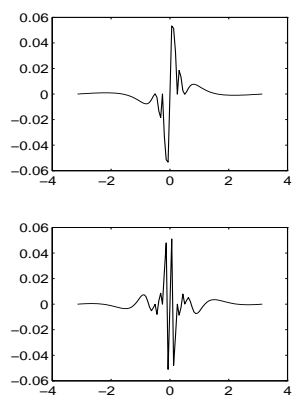

B: A "shifted" version of A

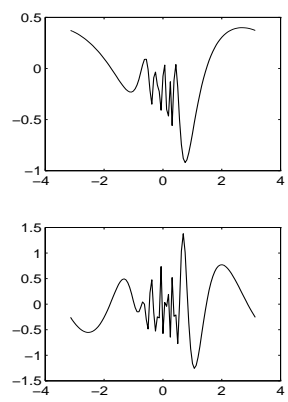

\section{Symmetry}

We have observed in several of the examples that the real part of the scaling and wavelet functions were symmetric while the imaginary parts showed an antisymmetric property. We can explain this as follows. Suppose that the measure $\mu$ is real and symmetric in the sense that $\mu(S)=\mu(\bar{S})$ where $S=\left\{e^{i \theta}: 0 \leq \theta_{0} \leq \theta \leq \theta_{1} \leq \pi\right\}$ is an arc of the upper half of the unit circle and $\bar{S}$ is the corresponding arc on the lower half circle: $\bar{S}=\{z: \bar{z} \in S\}$. Moreover assume that the poles $1 / \bar{\alpha}_{k}$ are chosen symmetrically, that is the poles used in $\mathcal{L}_{n}$ are either real or they appear in complex conjugate pairs. In that case the symmetry that was observed will take place.

THEOREM 11.1. Under the above conditions about symmetry of the measure and of the poles, the kernels $k_{2^{s}}(z, 1)$ and therefore also the kernels $l_{2^{s}}(z, 1)=$ $k_{2^{s+1}}(z, 1)-k_{2^{s}}(z, 1)$ have a symmetric real part and an antisymmetric imaginary part.

Proof. Suppose $n=2^{s}$. We first observe that $k_{n}(z, w)=\sum_{k=0}^{n} f_{k}(z) \overline{f_{k}(w)}$ where $\left\{f_{k}\right\}_{k=0}^{n}$ is any basis of orthogonal functions such that $\mathcal{L}_{n}=\operatorname{span}\left\{f_{k}: k=\right.$ $0, \ldots, n\}$. Now consider the measure $\mathrm{d} \mu_{n}(t)=\mathrm{d} \mu(t) /\left|\pi_{n}(t)\right|^{2}$ where $t=e^{i \theta}$ and $\pi_{n}(t)=\prod_{k=1}^{n}\left(1-\bar{\alpha}_{k} t\right)$. Define the polynomials $q_{k}, k=0,1, \ldots, n$ by orthogonalizing the functions $\left\{1, z, \ldots, z^{n}\right\}$ with respect to $\mu_{n}$. Because $\mu_{n}$ is real and symmetric on $\mathbb{T}$, the coefficients of the $p_{k}$ will be real. Hence $p_{k}(\bar{z})=\overline{p_{k}(z)}$. In particular $p_{k}(1) \in \mathbb{R}$. The reproducing kernel $\tilde{k}_{n}(z, 1)=\sum_{k=0}^{n} p_{k}(z) \overline{p_{k}(1)}$ for the polynomial space $\Pi_{n}$ with respect to $\mu_{n}$ will thus satisfy $\tilde{k}_{n}(\bar{z}, 1)=\overline{\tilde{k}_{n}(z, 1)}$. Furthermore, because of the symmetry of the $\alpha_{k}$, it holds that $\pi_{n}(\bar{z})=\overline{\pi_{n}(z)}$. The theorem now follows by observing that the kernel $\tilde{k}_{n}(z, 1)$ can be transformed into a reproducing kernel for $\mathcal{L}_{n}$ with respect to $\mu$ by setting $k_{n}(z, 1)=\tilde{k}_{n}(t, 1) /\left[\pi_{n}(t) \overline{\pi_{n}(1)}\right]$ and obviously $k_{n}(\bar{z}, 1)=\overline{k_{n}(z, 1)}$. 


\section{Computations}

We have given a recurrence for the reproducing kernels in Section 5. It was however not applicable in its forward form when $w \in \mathbb{T}$. There is however a backward algorithm, which is in fact the generalized Schur algorithm of NevanlinnaPick. One starts by computing the Riesz-Herglotz transform $\Omega$ of the measure $\mu$ and from this one computes a Schur function (bounded by 1 in modulus and analytic in $\mathbb{D}$ )

$$
\Gamma(z, w)=\frac{\Omega(z)-\Omega(w)}{\Omega(z)+\Omega(w)}
$$

where $z$ is the variable and $w$ a parameter such that $\Gamma(w, w)=0$. Given the points $\left\{\alpha_{k}\right\}$, one can compute the $\rho_{k}$ and $\gamma_{k}$ of the recurrence by the following algorithm. Set $\Gamma_{0}=\Gamma$, and compute for $k=1,2, \ldots$ the transforms $\Gamma_{k}=\mathcal{T}_{k}\left(\Gamma_{k-1}\right)$ where $\mathcal{T}_{k}=\mathcal{T}_{1 k} \circ \mathcal{T}_{2 k} \circ \mathcal{T}_{3 k}$ is defined by

$$
\begin{aligned}
& \mathcal{T}_{1 k}: \Gamma_{k-1} \mapsto \Gamma_{k}^{\prime}=\frac{\Gamma_{k-1}-\gamma_{k}}{1-\bar{\gamma}_{k} \Gamma_{k-1}}, \quad \gamma_{k}=\Gamma_{k-1}\left(\alpha_{k}, w\right) \\
& \mathcal{T}_{2 k}: \Gamma_{k}^{\prime} \mapsto \Gamma_{k}^{\prime \prime}=\gamma_{k}^{\prime} / \zeta_{k}, \\
& \mathcal{T}_{3 k}: \Gamma_{k}^{\prime \prime} \mapsto \Gamma_{k}=\frac{\Gamma_{k}^{\prime \prime}-\rho_{k}}{1-\bar{\rho}_{k} \Gamma_{k}^{\prime \prime}}, \quad \rho_{k}=\Gamma_{k}^{\prime \prime}(w, w) .
\end{aligned}
$$

The $\gamma_{k}$ 's and $\rho_{k}$ 's are the parameters that are needed in the recurrence relation of the reproducing kernels. So, given the values of $\Omega$ at the points $\alpha_{k}$, it is possible to generate the parameters $\rho_{k}$ and $\gamma_{k}$. However, if some (or all) of the points $\alpha_{k}$ coincide, then one has to provide the values of derivatives of $\Omega$ in these points and to cover the general case, it is rather difficult to write a tractable algorithm.

A similar argument holds for a Schur-type algorithm to compute the the parameters $\lambda_{k}$ in the recurrence relations for the ORF as given in Theorem 5.1.

In this text, we were mainly interested in experiments to generate plots for wavelets and see what the influence was of the the introduction of the poles $\alpha_{k}$, we have chosen to use the forward recursion for the ORF for some chosen $\alpha_{k}$ 's and $\lambda_{k}$ 's and from there compute the reproducing kernels. It is then however not easy to find what the underlying measure is. If the $\lambda_{k}$ are all in $\mathbb{T}$, then the existence of a positive measure is guaranteed by the Favard theorem [BGVHN92]. There are several theorems available describing the convergence of the kernels inside or outside the unit disk, but there are however practically no theorems to describe the convergence of the kernels on $\mathbb{T}$.

We do have the following direct result which is provided by Máté-Nevai-Totik [MNT91, Theorem 1].

THEOREM 12.1. Let all $\alpha_{k}=0$, so that we are in the polynomial case. Let $\phi_{k}$ be the orthonormal polynomials with respect to a finite positive Borel measure $\mathrm{d} \mu$ supported on $\mathbb{T}$. Define $k_{n}(t, t)=\sum_{k=0}^{n}\left|\phi_{k}(t)\right|^{2}, t \in \mathbb{T}$. Then if (Szegö's condition)

$$
\int_{-\pi}^{\pi} \log \mu^{\prime}(t) \mathrm{d} \theta>-\infty, \quad t=e^{i \theta}
$$

it holds that

$$
\lim _{n \rightarrow \infty} \frac{n}{k_{n}(t, t)}=\mu^{\prime}(t)
$$

for almost every $t \in \mathbb{T}$. 
We do not know of a proof which generalizes this to the rational case. We hope to provide such a proof in a future paper.

The previous theorem supposes however that $\log \mu^{\prime}$ is does not vanish on an interval of positive Lebesgue measure. For examples where Szegő's condition does not hold, we did observe in our experiments that it does not give the convergence hoped for. Also, convergence is relatively slow, which means that a large $n$ is needed before convergence occurs, so that one may run into numerical difficulties before the computation is complete.

There is however an alternative which is not completely proved either but it turns out to be numerically performing better. We can motivate it as follows.

First recall that if Szegö's condition is satisfied then one may define the spectral factor

$$
\sigma(z)=\exp \left\{\frac{1}{4 \pi} \int_{\mathbb{T}} \frac{t+z}{t-z} \log w(t) \mathrm{d} \theta\right\}, \quad t=e^{i \theta}
$$

for $z \in \mathbb{D}$ and we normalize it such that $\sigma(0)>0$. Moreover it has a radial limit to $\mathbb{T}$ such that $|\sigma(t)|^{2}=\mu^{\prime}(t)$ a.e. (w.r.t. Lebesgue measure) on $\mathbb{T}$.

For the convergence of the kernels inside the disk we have for example (see [BGVHN98b] or [BGVHN90]).

THEOREM 12.2. With $K_{n}(t)=k_{n}(t, 0) / \sqrt{k_{n}(0,0)}$ we have

$$
\langle f, g\rangle=\int_{\mathbb{T}} f(t) \overline{g(t)} \mathrm{d} \mu(t)=\int_{\mathbb{T}} f(t) \overline{g(t)} \mathrm{d} \mu_{n}(t), \quad \forall f, g \in \mathcal{L}_{n}
$$

where

$$
\mathrm{d} \mu_{n}(t)=\frac{\mathrm{d} \theta}{2 \pi\left|K_{n}(t)\right|^{2}}, \quad t=e^{i \theta}
$$

Moreover, if Szegö's condition holds, and the Blasche product diverges, then $K_{n}(z)$ converges locally uniformly to $1 / \sigma(z)$ in $\mathbb{D}$.

Thus we may hope that plotting $\left|K_{n}(t)\right|^{-2}$ for $t \in \mathbb{T}$ and $n$ sufficiently large will give an idea about the underlying weight.

ExAmPle 12.1. The previous idea is used in Figure 6. We took all $\alpha_{k}=0$ and chose $\left|\lambda_{1}\right|=\left|\lambda_{2}\right|=0.4$ and all other $\lambda_{k}=0$. We have plotted $k_{8}(t, 1)$ and $l_{8}(t, 1)$ and their modulus. The rightmost pictures show the unit circle with the position of the $\alpha_{k}=0$ and below it, we have plotted the approximation for the weight. In the figure $\mathrm{B}$ below, we plotted $k_{8}(t, 1)$ and $l_{8}(t, 1)$ multiplied by the weight (pictures at the bottom).

Both of the methods given above give bad convergence when Szegö's condition is not satisfied. However, it can be experimentally verified that in those cases we do have convergence of the $(C, 1)$ Cesàro sums

$$
S_{n}(t)=\frac{1}{n+1} \sum_{j=0}^{n} K_{j}(t), \quad t \in \mathbb{T}
$$

to the weight function $w$ in $\mathrm{d} \mu(t)=w(t) \mathrm{d} \theta, t=e^{i \theta}$.

EXAmPle 12.2. We give one last example in Figure 7. Here we took $\alpha_{1}=0$, $\alpha_{k}=0.9$ for all $k \geq 2$ All other $\alpha_{k}$ are zero. For the reflection coefficients we took the same as in the previous example: $\left|\lambda_{1}\right|=\left|\lambda_{2}\right|=0.4$ and all other $\lambda_{k}=0$. By 
FiguRE 6. The real and imaginary part and the modulus of the functions $k_{8}(z, 1)$ (top) and $l_{8}(z, 1)$ (bottom) where all $\alpha_{k}=0$ and $\left|\lambda_{1}\right|=\left|\lambda_{2}\right|=0.4$ and all other $\lambda_{k}=0$. This corresponds to a weight which is plotted in the lower right corner. In Figure B, we plotted the same functions (real and imaginary parts and modulus) multiplied by the weight.

A: $k_{8}(z, 1)$ and $l_{8}(z, 1)$


B: Same functions as in A, multiplied by the weight
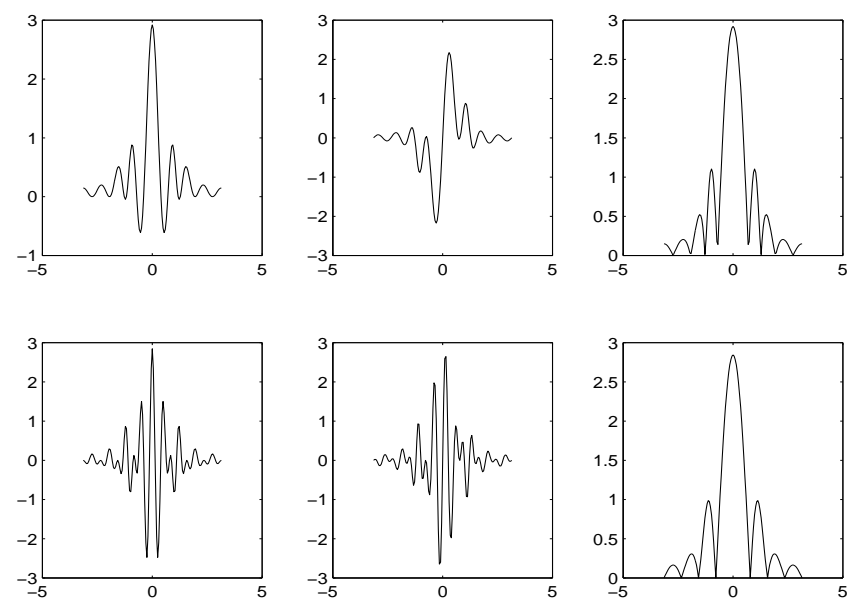

comparing with the Figure 6, one can notice the influence of the poles. The weight function is practically unchanged, but the wavelets are much more locallized.

\section{Conclusion}

We have given a rational generalization of the polynomial wavelets of Fischer and Prestin [FP97] and translated it to the orthogonal rational functions on the 
FiguRE 7. The real and imaginary part and the modulus of the functions $k_{8}(z, 1)$ (top) and $l_{8}(z, 1)$ (bottom) where all $\alpha_{k}=0.9$, except $\alpha_{1}=0$, and $\left|\lambda_{1}\right|=\left|\lambda_{2}\right|=0.4$ and all other $\lambda_{k}=0$. This corresponds to a weight which is plotted in the lower right corner. In Figure B, we plotted the same functions (real and imaginary parts and modulus) multiplied by the weight.

A: $k_{8}(z, 1)$ and $l_{8}(z, 1)$
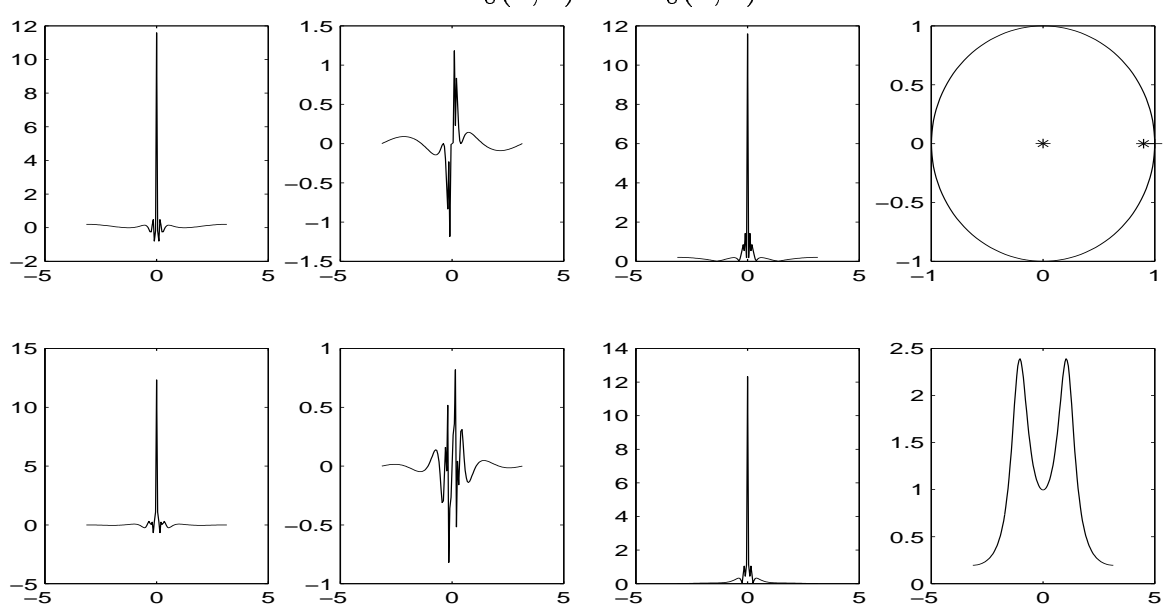

B: Same functions as in A, multiplied by the weight
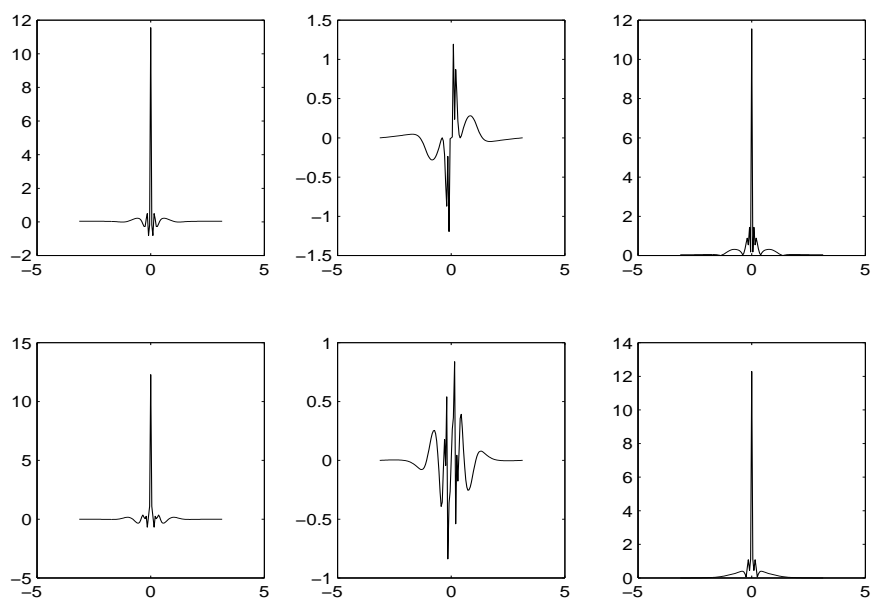

unit circle. The continued fraction-like recurrences and quadrature formulas related to these orthogonal rational functions play a central role.

This results in complex wavelets which can be influenced by the choice of poles near the unit circle.

When one wants to apply this to real $2 \pi$-periodic functions, the wavelets being complex is a drawback. Our theory can be generalized to orthogonal rational functions where the points $\alpha_{k}$ are chosen in a special way like in [BGVHN94, 
GVSLN96] where the orthogonal rational functions are obtained by orthogonalization of the sequence $\left\{B_{n}^{-1}, B_{n-1}^{-1}, \ldots, B_{1}^{-1}, 1, B_{1}, \ldots, B_{n-1}, B_{n}\right\}$.

\section{References}

[Ach56] N.I. Achieser, Theory of approximation, Frederick Ungar Publ. Co., New York, 1956.

[Aro50] N. Aronszajn, Theory of reproducing kernels, Trans. Amer. Math. Soc. 68 (1950), $337-404$.

[BGV98] A. Bultheel and P. González-Vera, Rational wavelets on the real line, Numer. Funct. Anal. Optim. (1998), Submitted.

[BGVHn90] A. Bultheel, P. González-Vera, E. Hendriksen, and O. Njåstad, A Szegő theory for rational functions, Technical Report TW131, Department of Computer Science, K.U. Leuven, May 1990.

[BGVHN92] A. Bultheel, P. González-Vera, E. Hendriksen, and O. Njåstad, A Favard theorem for orthogonal rational functions on the unit circle, Numer. Algorithms 3 (1992), 81-89.

[BGVHN94] A. Bultheel, P. González-Vera, E. Hendriksen, and O. Njåstad, Quadrature formulas on the unit circle based on rational functions, J. Comput. Appl. Math. 50 (1994), 159-170.

[BGVHN98a] A. Bultheel, P. González-Vera, E. Hendriksen, and O. Njåstad, Continued fractions and orthogonal rational functions, Orthogonal Functions, Moment Theory and Continued Fractions: Theory and Applications (W.B. Jones and A.S. Ranga, eds.), Marcel Dekker, 1998, To appear.

[BGVHN98b] A. Bultheel, P. González-Vera, E. Hendriksen, and O. Njåstad, Orthogonal rational functions, Cambridge University Press, 1998, (To appear).

[Bre90] C. Brezinski, A direct proof of the Christoffel-Darboux identity and its equivalence to the recurrence relationship, J. Comput. Appl. Math. 32 (1990), 17-25.

[Dav63] P.J. Davis, Interpolation and approximation, Blaisdell, New York, 1963, Reprint: Dover, 1975.

[Djr90] M.M. Djrbashian, A survey on the theory of orthogonal systems and some open problems, Orthogonal polynomials: Theory and practice (Boston) (P. Nevai, ed.), Series C: Mathematical and Physical Sciences, vol. 294, NATO-ASI, Kluwer Academic Publishers, 1990, pp. 135-146.

[Don74] W.F. Donoghue Jr., Monotone matrix functions and analytic continuation, Springer, Berlin, 1974.

[FP97] B. Fischer and J. Prestin, Wavelets based on orthogonal polynomials, Math. Comp. 66 (1997), 1593-1618.

[GVSLN96] P. González-Vera, J.C. Santos-Leon, and O. Njåstad, Some results about numerical quadrature on the unit circle, Adv. Comput. Math. 5 (1996), 297-328.

[Mes62] H. Meschkowski, Hilbertsche Räume mit Kernfunktion, Springer, Berlin, 1962.

[MNT91] A. Máté, P. Nevai, and V. Totik, Szegö's extremum problem on the unit circle, Ann. of Math. 134 (1991), 433-453.

[Swe98] W. Sweldens, The lifting scheme: A construction of second generation wavelets, SIAM J. Math. Anal. 29 (1998), 511-548.

[Sze67] G. Szegö, Orthogonal polynomials, 3rd ed., Amer. Math. Soc. Colloq. Publ., vol. 33, Amer. Math. Soc., Providence, Rhode Island, 1967, First edition 1939.

Department of Computer Science, K.U.Leuven, Leuven, Belgium

E-mail address: Adhemar.Bultheel@cs.kuleuven. ac.be

$U R L:$ http://www.cs.kuleuven.ac.be/ ${ }^{\sim}$ ade/

Department Análisis Math., Univ. La Laguna, Tenerife, Spain

E-mail address: pglez@ull.es 